\title{
Variational boundary conditions based on the Nitsche method for fitted and unfitted isogeometric discretizations of the mechanically coupled Cahn-Hilliard equation
}

\author{
Ying Zhao ${ }^{\mathrm{a}, \mathrm{b}, *}$, Dominik Schillinger ${ }^{\mathrm{c}}$, Bai-Xiang $\mathrm{Xu}^{\mathrm{b}}$ \\ ${ }^{a}$ Graduate School of Computational Engineering, Technische Universität Darmstadt, Germany \\ ${ }^{b}$ Mechanics of Functional Materials Division, Dept. of Materials Science, Technische Universität Darmstadt, Germany \\ ${ }^{c}$ Department of Civil, Environmental, and Geo- Engineering, University of Minnesota, Minneapolis, USA
}

\begin{abstract}
The primal variational formulation of the fourth-order Cahn-Hilliard equation requires $\mathscr{C}^{1}$ continuous finite element discretizations, e.g., in the context of isogeometric analysis. In this paper, we explore the variational imposition of essential boundary conditions that arise from the thermodynamic derivation of the Cahn-Hilliard equation in primal variables. Our formulation is based on the symmetric variant of Nitsche's method, does not introduce additional degrees of freedom and is shown to be variationally consistent. In contrast to strong enforcement, the new boundary condition formulation can be naturally applied to any mapped isogeometric parametrization of any polynomial degree. In addition, it preserves full accuracy, including higher-order rates of convergence, which we illustrate for boundary-fitted discretizations of several benchmark tests in one, two and three dimensions. Unfitted Cartesian B-spline meshes constitute an effective alternative to boundary-fitted isogeometric parametrizations for constructing $\mathscr{C}^{1}$-continuous discretizations, in particular for complex geometries. We combine our variational boundary condition formulation with unfitted Cartesian B-spline meshes and the finite cell method to simulate chemical phase segregation in a composite electrode. This example, involving coupling of chemical fields with mechanical stresses on complex domains and coupling of different materials across complex interfaces, demonstrates the flexibility of variational boundary conditions in the context of higher-order unfitted isogeometric discretizations.
\end{abstract}

Keywords: Variational boundary conditions, the Nitsche method, Cahn-Hilliard equation, Isogeometric analysis, Finite cell method, Composite electrode

\section{Introduction}

The Cahn-Hilliard equation is a fourth-order partial differential equation that was proposed as the evolution equation for a conserved order parameter [1, 2], preserved during phase trans-

\footnotetext{
${ }^{*}$ Corresponding author; Graduate School of Computational Engineering, Technische Universität Darmstadt, Dolivostrasse 15, 64293 Darmstadt, Germany

Email addresses: zhao@gsc.tu-darmstadt.de (Ying Zhao), dominik@umn . edu (Dominik Schillinger), xu@mfm.tu-darmstadt.de (Bai-Xiang Xu)
}

Preprint submitted to fournal of Computational Physics

March 10, 2017 
formation in the absence of an external flux. Its primal variational formulation contains a secondorder differential operator, which requires globally $\mathscr{C}^{1}$-continuous basis functions, when a finite element discretiatzion is sought. Alternative strategies include mixed formulations, where the Cahn-Hilliard equation is rewritten into two coupled equations, and discontinuous Galerkin methods. A mixed formulation is based on the introduction of an additional variable, which can be a nonlocal species concentration [3], or the chemical potential [4]. Discontinous Galerkin methods for the Cahn-Hilliard equation require flux formulations that enforce the $\mathscr{C}^{1}$-continuity in a weak sense [5-7] . Both approaches reduce the requirement of continuity of the elements, so that standard $\mathscr{C}^{0}$-continuous basis functions can be used, but lead to larger discrete systems due to additional degrees of freedom.

Isogeometric analysis [8] is another alternative that has attracted increasing attention in the context of Cahn-Hilliard based simulation over the last years [9-16]. Isogeometric analysis uses higher-order continuous spline basis functions and can therefore treat higher-order differential operators in a straightforward fashion. In isogeometric discretizations of the primal formulation, however, special attention has to be paid to enforce an essential boundary condition that arises from the thermodynamic derivation of the Cahn-Hilliard equation and states that the normal gradient of the concentration must be zero.

This condition has turned out to be difficult to enforce. In Gomez et al. [9], this problem is avoided by imposing periodic boundary conditions with periodic B-spline basis functions. Employing quadratic shape functions in rectangular/cuboid grids, Liu et al. [10] imposed this condition by setting two consecutive boundary control values to be equal. Dalcin et al. [14] developed an "unclamping algorithm" to adapt higher-order open knot vectors to the desired continuity across the boundary, that can be applied to enforce periodic boundary conditions. However, these variants of strongly imposing essential boundary conditions are often limited by constraints on the geometric parametrization, in terms of the polynomial degree, a Cartesian grid, or the presence of control axes that are perpendicular to the boundary. Alternatively, Anders et al. [16] and Zhao et al. [11] imposed this boundary condition using Lagrange multipliers, which is less restrictive in terms of isogeometric discretizations and their polynomial degree, but again requires additional degrees of freedom.

The first objective of this paper is to explore a different boundary condition formulation for the Cahn-Hilliard equation that is free of geometric constraints, accommodates arbitrary poly35 nomial degrees and preserves higher-order accuracy and convergence, and directly generalizes to unfitted discretizations and embedded surfaces. It is based on the original idea of Nitsche who developed a variationally consistent penalty method for weakly enforcing Dirichlet boundary conditions that is symmetric and free of auxiliary fields [17]. The variational consistency of the symmetric Nitsche method allows the reinterpretation of the penalty parameter as a mesh dependent stabilization parameter that needs to be chosen sufficiently large in order to maintain stability of the bilinear form [18-21]. The second objective of this paper is to combine the variational boundary condition formulation with unfitted B-spline discretizations and the finite cell method [22, 23]. The resulting framework, enabled by variational boundary conditions, significantly facilitates the analysis of Cahn-Hilliard based models on complex irregular geometries in three dimensions.

Our paper is organized as follows: In Section 2, we first review the derivation of the Cahn- 
Hilliard equation in a thermodynamic context, with particular emphasis on the additional essential boundary conditions. We then derive the variational Nitsche formulation, building on related work for other fourth-order differential equations [24, 25]. As a special case, the formulation for periodic boundary conditions is discussed. In Section 3, a brief overview of numerical techniques which are used in this paper is given. In particular, isogeometric analysis with fitted geometry and the finite cell method based on isogeometric discretizations are reviewed. In Section 4 , several numerical examples in one, two and three dimensions are considered in the context of boundary-fitted isogeometric analysis that demonstrate the performance of the new formulation in terms of accuracy and geometric flexibility. In Section 5 , we consider the charging process of a particle in the composite electrode of a lithium-ion battery [26, 27]. We first review the governing equations in the composite electrode, and then demonstrate accuracy and flexibility of the resulting framework for solving the primal formulation of the Cahn-Hilliard equation on irregular particle geometries with higher-order continuous basis functions. Section 6 puts our variational formulation and numerical results into perspective and motivates future work.

\section{The Cahn-Hilliard equation and its variational formulation with the Nitsche method}

We begin with a review of the Cahn-Hilliard equation in a thermodynamics context, the associated initial boundary value problem and its variational formulation. In particular, a corresponding Nitsche extension for weakly enforcing essential boundary conditions is introduced, including the periodic case.

\subsection{Derivation of the Cahn-Hilliard equation from thermodynamics}

Following the theory of Cahn and Hilliard [1,2], a binary solution system is considered, where the evolution of the non-uniform mole fraction (or normalized concentration) of one component $c$ over time $t$ is given by

$$
\frac{\partial c}{\partial t}=-\nabla \cdot \boldsymbol{j}
$$

where the flux $j$ is defined as

$$
\boldsymbol{j}=-M_{c} \boldsymbol{\nabla} \mu .
$$

Here, $M_{c}$ represents a degenerated mobility term in the limit of $c=0$ and $c=1$, expressed as $M=c(1-c)$ in this paper. The chemical potential is defined as the variational derivative of the total free energy with respect to the species concentration:

$$
\mu=\delta_{c} \Psi=\delta_{c} \int_{\Omega}\left(\psi_{c}+\frac{1}{2} \kappa|\nabla c|^{2}\right) \mathrm{d} \Omega
$$

where the bulk chemical energy density $\psi_{c}$ prescribes a double-well function, allowing for the coexistence of two phases. The term containing $\nabla c$ represents an energetic penalty for the phase interface and $\kappa$ is a parameter related to the interfacial thickness. In the limit of $\kappa \rightarrow 0$, the interfacial thickness approaches 0 . For a detailed discussion of the theory that forms the basis for determining the interfacial thickness, interested readers are referred to for example our previous 
work [11]. Since

$$
\delta \Psi=\delta \int_{\Omega}\left(\psi_{c}+\frac{1}{2} \kappa|\nabla c|^{2}\right) \mathrm{d} \Omega=\int_{\Omega}\left(\frac{\mathrm{d} \psi_{c}}{\mathrm{~d} c}-\kappa \Delta c\right) \delta c \mathrm{~d} \Omega+\int_{\partial \Omega} \kappa \boldsymbol{\nabla} c \cdot \boldsymbol{n} \delta c \mathrm{~d} \Gamma,
$$

80

the chemical potential is derived as

$$
\mu=\frac{\mathrm{d} \psi_{c}}{\mathrm{~d} c}-\kappa \Delta c
$$

under the condition that $\kappa \boldsymbol{\nabla} c \cdot \boldsymbol{n}=0$ on the entire boundary $\partial \Omega$. In this paper, we consider a regular solution model, the details of which can be found in the book by Guggenheim [28]. The bulk chemical free energy $\psi_{c}$ is given as

$$
\psi_{c}=c \ln c+(1-c) \ln (1-c)+\chi c(1-c),
$$

where the positive $\chi$ is an interchange-energetic parameter to indicate a non-ideal mixture. $\chi>2$ gives a non-convex $\psi_{c}$, allowing for two coexisting phases. The corresponding flux $\boldsymbol{j}$ is then expressed as

$$
\boldsymbol{j}=-\{[1-2 \chi c(1-c)] \boldsymbol{\nabla} c-c(1-c) \boldsymbol{\nabla}(\kappa \Delta c)\} .
$$

\subsection{Strong form of the initial boundary value problem}

The above discussion gives rise to the following problem in strong form: find the concentration $c: \Omega \times(0, \mathrm{~T}) \rightarrow \mathrm{R}$ such that

$$
\frac{\partial c}{\partial t}=\nabla \cdot\{[1-2 \chi c(1-c)] \nabla c-c(1-c) \nabla(\kappa \Delta c)\} \quad \text { in } \quad \Omega \times(0, \mathrm{~T})
$$

satisfying the set of boundary conditions

$$
\begin{array}{ll}
c=\hat{c} & \text { on } \Gamma_{c} \times(0, \mathrm{~T}), \\
\{[1-2 \chi c(1-c)] \boldsymbol{\nabla} c-c(1-c) \boldsymbol{\nabla}(\kappa \Delta c)\} \cdot \boldsymbol{n}=\hat{j} & \text { on } \Gamma_{j} \times(0, \mathrm{~T}), \\
\kappa \boldsymbol{\nabla} c \cdot \boldsymbol{n}=0 & \text { on } \partial \Omega \times(0, \mathrm{~T})
\end{array}
$$

and the initial condition

$$
c(\boldsymbol{x}, 0)=c_{0}(\boldsymbol{x}) \quad \text { on } \Omega .
$$

The first two boundary terms denote Dirichlet and Neumann conditions, with a given concentration $\hat{c}$ and boundary flux $\hat{j}$. The corresponding Dirichlet and Neumann boundaries $\Gamma_{c}$ and $\Gamma_{j}$ are complementary subsets of the complete domain boundary $\partial \Omega$. Eq. (11) is an additional essential boundary condition that holds on $\partial \Omega$. Motivated in (4) and (5), it ensures consistency with the variational derivation. 


\subsection{Nitsche's method for the Cahn-Hilliard equation}

In the spirit of Nitsche's method for fourth-order problems [5, 24, 25], the following variational formulation with weakly enforced boundary conditions for the Cahn-Hilliard equation can be derived: find $c \in \mathcal{S} \times(0, \mathrm{~T})$ such that for all $\delta c \in \mathcal{V} \times(0, \mathrm{~T})$

$$
\begin{aligned}
& \int_{\Omega} \frac{\partial c}{\partial t} \delta c \mathrm{~d} \Omega+\int_{\Omega}[1-2 \chi c(1-c)] \boldsymbol{\nabla} c \cdot \boldsymbol{\nabla} \delta c \mathrm{~d} \Omega+\int_{\Omega}(1-2 c) \kappa \Delta c \boldsymbol{\nabla} c \cdot \boldsymbol{\nabla} \delta c \mathrm{~d} \Omega \\
& +\int_{\Omega} c(1-c) \kappa \Delta c \Delta \delta c \mathrm{~d} \Omega-\int_{\Gamma_{c}}\{[1-2 \chi c(1-c)] \boldsymbol{\nabla} c-c(1-c) \boldsymbol{\nabla}(\kappa \Delta c)\} \cdot \boldsymbol{n} \delta c \mathrm{~d} \Gamma \\
& -\int_{\Gamma_{c}} c \boldsymbol{\nabla}(\kappa \Delta \delta c) \cdot \boldsymbol{n} \mathrm{d} \Gamma+\alpha_{1} \int_{\Gamma_{c}} c \delta c \mathrm{~d} \Gamma-\int_{\partial \Omega} \kappa c(1-c) \Delta c(\boldsymbol{\nabla} \delta c \cdot \boldsymbol{n}) \mathrm{d} \Gamma \\
& -\int_{\partial \Omega} \kappa c(1-c)(\boldsymbol{\nabla} c \cdot \boldsymbol{n}) \Delta \delta c \mathrm{~d} \Gamma+\alpha_{2} \int_{\partial \Omega}(\boldsymbol{\nabla} c \cdot \boldsymbol{n})(\boldsymbol{\nabla} \delta c \cdot \boldsymbol{n}) \mathrm{d} \Gamma \\
& =\int_{\Gamma_{j}} \hat{j} \delta c \mathrm{~d} \Gamma-\int_{\Gamma_{c}} \hat{c} \boldsymbol{\nabla}(\kappa \Delta \delta c) \cdot \boldsymbol{n} \mathrm{d} \Gamma+\alpha_{1} \int_{\Gamma_{c}} \hat{c} \delta c \mathrm{~d} \Gamma .
\end{aligned}
$$

The spaces spanned by the basis functions for approximation and weighting are $\mathcal{S}=H^{2}$ and $\mathcal{V}=H^{2}$, where $H^{2}$ is the space of square integrable functions with square integrable first and second derivatives [24]. The stabilization parameters $\alpha_{1}$ and $\alpha_{2}$ need to be chosen large enough as to guarantee stability of (13). The terms defined on the Dirichlet boundary $\Gamma_{c}$ and associated stabilization with $\alpha_{1}$ weakly enforce a given concentration $\hat{c}$. The terms defined on the complete domain boundary $\partial \Omega$ and associated stabilization with $\alpha_{2}$ weakly enforce that the normal derivative is zero.

To show the consistency of the variational form (13) with the strong form of the boundary value problem Eq. (8)-(11), we apply repeated integration by parts to Eq. (13). This results in

$$
\begin{aligned}
& \int_{\Omega} \frac{\partial c}{\partial t} \delta c \mathrm{~d} \Omega-\int_{\Omega} \boldsymbol{\nabla} \cdot\{[1-2 \chi c(1-c)] \boldsymbol{\nabla} c-c(1-c) \boldsymbol{\nabla}(\kappa \Delta c)\} \delta c \mathrm{~d} \Omega \\
& +\int_{\Gamma_{j}}(\{[1-2 \chi c(1-c)] \boldsymbol{\nabla} c-c(1-c) \boldsymbol{\nabla}(\kappa \Delta c)\} \cdot \boldsymbol{n}-\hat{j}) \delta c \mathrm{~d} \Gamma \\
& -\int_{\Gamma_{c}}(c-\hat{c}) \boldsymbol{\nabla}(\kappa \Delta \delta c) \cdot \boldsymbol{n} \mathrm{d} \Gamma+\alpha_{1} \int_{\Gamma_{c}}(c-\hat{c}) \delta c \mathrm{~d} \Gamma \\
& -\int_{\partial \Omega} \kappa c(1-c)(\boldsymbol{\nabla} c \cdot \boldsymbol{n}) \Delta \delta c \mathrm{~d} \Gamma+\alpha_{2} \int_{\partial \Omega}(\boldsymbol{\nabla} c \cdot \boldsymbol{n})(\boldsymbol{\nabla} \delta c \cdot \boldsymbol{n}) \mathrm{d} \Gamma=0,
\end{aligned}
$$

With the argument that (14) must hold for arbitrary weighting functions $\delta c$, we can identify the strong form of the boundary value problem Eq. (8)-(11).

Remark: In the scope of this work, the stabilization parameters $\alpha_{1}$ and $\alpha_{2}$ are chosen empirically. They can also be estimated by global or element-wise eigenvalue problems as shown for example in [24, 25, 29].

\subsection{Periodic boundary conditions}

A typical situation is the evolution of a concentration field from a random initial state on a rectangular or cuboid domain under periodic boundary conditions. Therefore, we extend the Nitsche formulation to the case of periodic boundary conditions. 


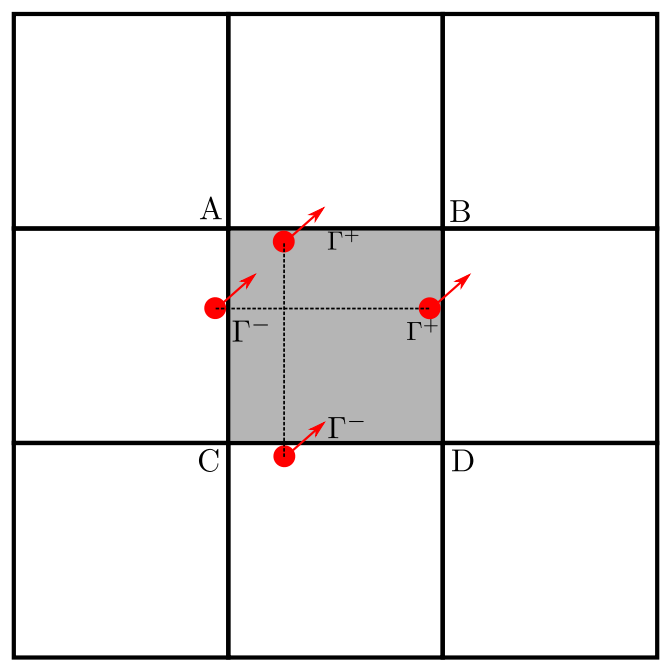

FIGURE 1: Schematic of the periodic box (shaded area). For simplicity, a square is considered. $\overline{A C}, \overline{C D}$ are entry boundaries $\Gamma^{-}$, and $\overline{B D}, \overline{A B}$ are the corresponding exit boundaries $\Gamma^{+}$.

For simplicity, we restrict the periodic box to be a square, as shown in Figure 1. Periodic boxes of other shapes can be imposed in the similar manner. Let $\Gamma^{-}$and $\Gamma^{+}$denote the entry and exit boundary of a periodic box $\Omega$, which satisfies $\Gamma^{-} \cup \Gamma^{+}=\partial \Omega$ and $\Gamma^{-} \cap \Gamma^{+}=\emptyset \cdot \boldsymbol{x}^{-} \in \Gamma^{-}$ and $\boldsymbol{x}^{+} \in \Gamma^{+}$are two matching boundary points with constituents $(\cdot)^{-}$and $(\cdot)^{+}$, respectively. We note that we will use the condition

$$
\int_{\Gamma^{-}}(\cdot) \mathrm{d} \Gamma=\int_{\Gamma^{+}}(\cdot) \mathrm{d} \Gamma
$$

assuming that $\Gamma^{-}$and $\Gamma^{+}$are geometrically symmetric.

We define the jump operator $\llbracket \cdot \rrbracket$ as

$$
\begin{array}{lll}
\llbracket a \rrbracket=a^{-}-a^{+}, & & a \in \mathrm{R}, \\
\llbracket \boldsymbol{a} \rrbracket=\boldsymbol{a}^{-} \cdot \boldsymbol{n}^{-}+\boldsymbol{a}^{+} \cdot \boldsymbol{n}^{+}, & & \boldsymbol{a} \in \mathrm{R}^{d}, d=2,3
\end{array}
$$

and the average operator $\langle\cdot\rangle$ as

$$
\begin{array}{ll}
\langle a\rangle=\frac{1}{2}\left(a^{-}+a^{+}\right), & a \in R, \\
\langle\boldsymbol{a}\rangle=\frac{1}{2}\left(\boldsymbol{a}^{-} \cdot \boldsymbol{n}^{-}-\boldsymbol{a}^{+} \cdot \boldsymbol{n}^{+}\right), & \boldsymbol{a} \in \mathrm{R}^{d}, d=2,3 .
\end{array}
$$

The general set of boundary conditions Eq. (9)-(11) can then be specified to the following set of periodic boundary conditions in strong form:

$$
\begin{array}{ll}
\left.c\right|_{\Gamma^{-}}-\left.c\right|_{\Gamma^{+}}=c^{-}-c^{+}=\llbracket c \rrbracket=0 & \text { on } \partial \Omega \times(0, \mathrm{~T}), \\
\left.\boldsymbol{\nabla} c \cdot \boldsymbol{n}\right|_{\Gamma^{-}}+\left.\boldsymbol{\nabla} c \cdot \boldsymbol{n}\right|_{\Gamma^{+}}=\boldsymbol{\nabla} c^{-} \cdot \boldsymbol{n}^{-}+\boldsymbol{\nabla} c^{+} \cdot \boldsymbol{n}^{+}=\llbracket \boldsymbol{\nabla} c \rrbracket=0 & \text { on } \partial \Omega \times(0, \mathrm{~T}),
\end{array}
$$




$$
\begin{array}{ll}
\left.\kappa \Delta c\right|_{\Gamma^{-}}-\left.\kappa \Delta c\right|_{\Gamma^{+}}=\kappa \Delta c^{-}-\kappa \Delta c^{+}=\llbracket \kappa \Delta c \rrbracket=0 & \text { on } \partial \Omega \times(0, \mathrm{~T}), \\
\left.\boldsymbol{j} \cdot \boldsymbol{n}\right|_{\Gamma^{-}}+\left.\boldsymbol{j} \cdot \boldsymbol{n}\right|_{\Gamma^{+}}=\boldsymbol{j}^{-} \cdot \boldsymbol{n}^{-}+\boldsymbol{j}^{+} \cdot \boldsymbol{n}^{+}=\llbracket \boldsymbol{j} \rrbracket=0 & \text { on } \partial \Omega \times(0, \mathrm{~T}),
\end{array}
$$

where the flux $j$ is given by Eq. (7). The boundary condition Eq. (18) is enforced strongly by setting the two matching control values on the corresponding boundaries equal. Further, in analogy to equation Eq. (13), Eq. (19)-Eq. (21) are imposed weakly using the Nitsche method. The corresponding variational statement reads

$$
\begin{aligned}
& \int_{\Omega} \frac{\partial c}{\partial t} \delta c \mathrm{~d} \Omega+\int_{\Omega}[1-2 \chi c(1-c)] \nabla c \cdot \nabla \delta c \mathrm{~d} \Omega+\int_{\Omega}(1-2 c) \kappa \Delta c \boldsymbol{\nabla} c \cdot \boldsymbol{\nabla} \delta c \mathrm{~d} \Omega \\
& +\int_{\Omega} c(1-c) \kappa \Delta c \Delta \delta c \mathrm{~d} \Omega-\int_{\Gamma^{-}} c(1-c)\langle\kappa \Delta c\rangle \llbracket \nabla \delta c \rrbracket \mathrm{d} \Gamma \\
& -\int_{\Gamma^{-}} c(1-c) \llbracket \nabla c \rrbracket\langle\kappa \Delta \delta c\rangle \mathrm{d} \Gamma+\alpha_{2} \int_{\Gamma^{-}} \llbracket \nabla c \rrbracket \llbracket \nabla \delta c \rrbracket \mathrm{d} \Gamma=0,
\end{aligned}
$$

We again can show consistency with respect to the strong form Eq. (8) and Eq. (18)-(21) by repeatedly applying integration by parts on (22), which leads to

$$
\begin{aligned}
& \int_{\Omega} \frac{\partial c}{\partial t} \delta c \mathrm{~d} \Omega-\int_{\Omega} \boldsymbol{\nabla} \cdot\{[1-2 \chi c(1-c)] \nabla c-\kappa c(1-c) \nabla \Delta c\} \delta c \mathrm{~d} \Omega \\
& -\int_{\Gamma^{-}} \llbracket \boldsymbol{j} \rrbracket \delta c \mathrm{~d} \Gamma+\int_{\Gamma^{-}} c(1-c) \llbracket \kappa \Delta c \rrbracket\langle\boldsymbol{\nabla} \delta c\rangle \mathrm{d} \Gamma \\
& -\int_{\Gamma^{-}} c(1-c) \llbracket \nabla c \rrbracket\langle\kappa \Delta \delta c\rangle \mathrm{d} \Gamma+\alpha_{2} \int_{\Gamma^{-}} \llbracket \nabla c \rrbracket \llbracket \nabla \delta c \rrbracket \mathrm{d} \Gamma=0,
\end{aligned}
$$

Making again use of the argument that (23) must hold for arbitrary weighting functions $\delta c$, we can identify the strong form of the boundary value problem (8) and the periodic boundary conditions (19) $-(21)$.

\section{Discretization with fitted and unfitted isogeometric finite elements}

Before discussing its numerical performance, we outline the finite element techniques that we will use for discretizing the variational framework for the Cahn-Hilliard equation derived in the previous section. In the scope of the present work, we only provide a brief overview of the different components of the discretization technology, but add ample references that will guide the interested reader to the pertinent literature.

\subsection{Isogeometric analysis and higher-order continuous basis functions}

Isogeometric analysis [8, 30] is an isoparametric finite element method. It uses smooth and higher-order spline basis functions, ubiquitous in computer aided geometric design (CAD) to represent geometric objects, for the representation of the geometry and the approximation of solutions fields. The original objective of isogeometric analysis has been a better integration of $\mathrm{CAD}$ and finite element analysis. However, isogeometric analysis has turned out to offer significant additional benefits. For problems with smooth solutions, it exhibits increased accuracy and robustness on a per-degree-of-freedom basis [22, 31, 32]. Unlike $\mathscr{C}^{0}$-continuous basis functions, 


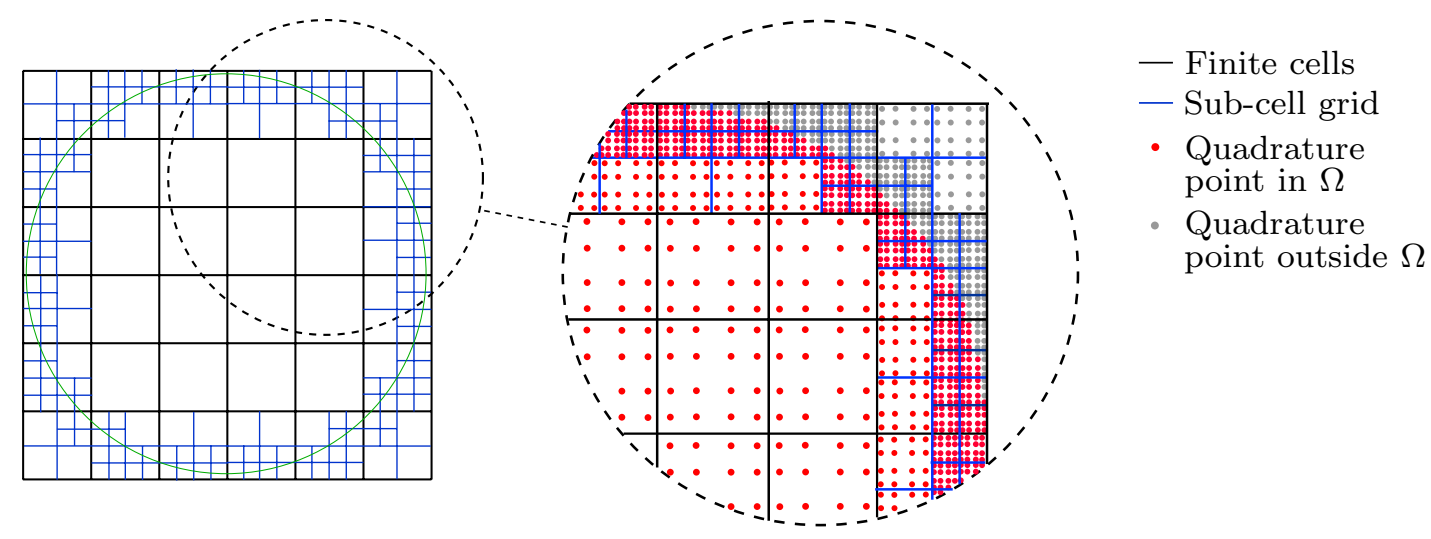

FIGURE 2: Recursive subdivision based quadrature in the finite cell method.

the higher modes of spline basis functions do not diverge with increasing degree, but achieve almost spectral accuracy that improves with degree [33-35]. Approximations of derivative fields are smooth and their degree can be adjusted to what is required by the primal variational formulation [9, 36, 37]. In the present paper, we exploit the higher-order continuity of splines for the direct discretiaztion of the primal variational form of the Cahn-Hilliard equation.

In this paper, fitted and unfitted isogeometric meshes based on quadratic and cubic B-splines and non-uniform rational B-splines (NURBS) are employed. For details on how to construct these basis functions and how to implement them efficiently in finite element analysis, the readers are referred for example to [30, 38-40]. Additional background on how to use isogeometric analysis in the context of the Cahn-Hilliard equations can be found for example in [9-13, 41].

\subsection{The finite cell method}

Unfitted finite element discretizations that do not conform to the boundaries of the physical domain require two fundamental components beyond standard finite element technology. First, they need to be able to enforce Dirichlet boundary conditions at embedded surfaces. In our context, the variational formulation of boundary conditions based on the Nitsche method in Eq. (13) naturally accommodates this requirement. Corresponding surface integrals do not depend on element boundaries and can be defined at any embedded surface that intersects the unfitted mesh. Second, unfitted discretizations rely on a quadrature method that is able to numerically evaluate surface and volume integrals in cut elements. For evaluating surface integrals in this paper, we simply introduce a fine triangulation of each embedded boundary. Each triangle serves as the basis for generating independent quadrature points, based on standard monomial rules [42, 43], each of which can be related to a specific element via its global coordinate. For volume quadrature in cut elements, we adopt the finite cell method.

The volume quadrature approach of the finite cell method employs composed Gauss quadrature, based on a hierarchical decomposition of the original element into quadrature sub-cells [44]. Figure 2 illustrates the concept for a circular domain, embedded in a Cartesian mesh. In a first step, each cut element is subdivided in sub-cells, constructed in the sense of a quadtree. Starting 
from the original cut element, each sub-cell is recursively queried whether it is cut by an embedded boundary. This query is usually based on a point location operation that determines whether characteristic points of the sub-cell are inside or outside the physical domain. If cut, the sub-cell is replaced by four equally spaced sub-cells of the next quadtree level. Partitioning is repeated until a predefined maximum depth is reached. Each sub-cell is equipped with $(p+1) \times(p+1)$ Gauss points, where $p$ is the polynomial degree of the basis. We note that in addition to standard Gauss point weights, a geometric weight is required that weights each point according to its quadtree level. All quadrature points that are located outside the physical domain are discarded.

To clearly distinguish between finite elements and quadrature sub-cells in Fugure 2, finite elements are plotted in black lines, while quadrature sub-cells are plotted in blue lines. We emphasize again that basis functions for the approximation of the solution fields are still defined exclusively on the black mesh, while the blue sub-cell structure exists only for defining adaptive quadrature points, aggregated around the geometric boundary. The recursive subdivision approach can be easily adjusted to one or three dimensions in the sense of binary or octrees, respectively. It can also be generalized easily to other element shapes such as tetrahedral [45, 46] elements.

The recursive subdivision approach is easy to implement, keeps a regular grid structure, and requires considerably less computational effort than non-adaptive schemes. The process of evaluating quadrature points constitutes a major computational cost, but is very well-suited for parallelization, which requires minimal communication and significantly speeds up finite cell computations. Due to the flexibility of its quadrature approach, the finite cell method can operate with almost any geometric model, ranging from boundary representations in computer aided geometric design to voxel representations obtained from medical imaging technologies. An important preliminary is the availability of an efficient point location query, which can be based on CAD tools or explicit voxelization of domains. A more detailed account of the technology along with a large number of application examples can be found in the recent review article by Schillinger and Ruess [23].

Schillinger et al. [22, 47] have combined the finite cell method with higher-order continuous B-spline basis functions for the analysis of volumetric structures based on trimmed spline surfaces. In this paper, we will demonstrate the flexibility of the finite cell method on Cartesian B-spline meshes for Cahn-Hilliard based simulations on complex geometries.

\subsection{Time integration and nonlinear solution}

For time integration of the Cahn-Hilliard equation, we employ a simple backward Euler method with adaptive time step control, where the time step is adjusted based on the number of iterations per time step. We define the minimum and maximum number of iterations as IMIN and IMAX, respectively. In addition, we also restrict the value of time between DTMIN and DTMAX. The time steps $d t_{\text {new }}$ are then adjusted automatically from the previous time step $d t_{\text {old }}$ based on the number of iterations $n_{\text {iter }}$ as

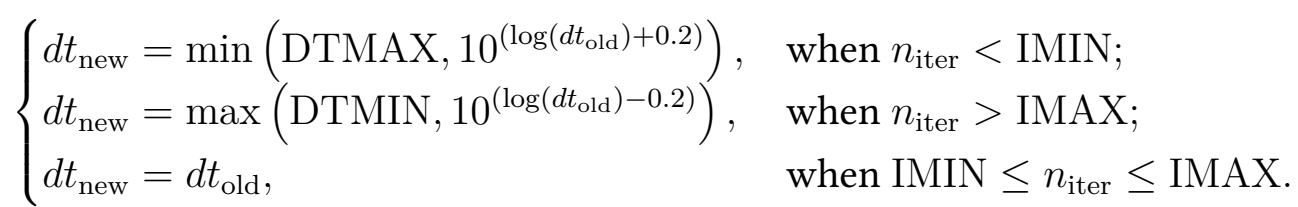


The nonlinear system is solved in each time step with a standard Newton-Raphson iteration scheme. We leverage corresponding existing routines implemented in the open-source finite element program FEAP [48] with isogeometric basis functions, to which we added the recursive subdivision based quadrature approach of the finite cell method, and the Nitsche extension for the Cahn-Hilliard equation.

\section{Weakly enforced boundary conditions for the Cahn-Hilliard equation on fitted iso- geometric parametrizations}

We first demonstrate the geometric flexibility and higher-order accuracy achieved with variationally formulated weak boundary conditions in the Cahn-Hilliard equation in the context of standard boundary-fitted isogeometric analysis. To this end, we consider several numerical examples in one, two and three dimensions.

\subsection{A $1 D$ bar with two coexisting phases}

The first example consists of a bar of length $\mathrm{L}=10$. The initial conditions are set as

$$
c(x, 0)= \begin{cases}0.2 & 0 \leq x \leq 5 \\ 0.8 & 5<x \leq 10\end{cases}
$$

The flux-free boundary conditions are given as

$$
\begin{array}{ll}
{[1-2 \chi c(1-c)] \frac{\partial c}{\partial x}-c(1-c) \kappa \frac{\partial^{3} c}{\partial x^{3}}=0,} & x=0, \mathrm{~L}, \quad t \in(0, \mathrm{~T}), \\
\kappa \frac{\partial c}{\partial x}=0, & x=0, \mathrm{~L}, \quad t \in(0, \mathrm{~T}) .
\end{array}
$$

We assume $\chi=2.5$, so that there are two homogeneous phases at equilibrium with concentrations $c_{\alpha}=0.1448$ and $c_{\beta}=0.8552$. The interfacial parameter is set as $\kappa=0.01$.

For a first impression, we compute the solution of the Cahn-Hilliard equation with weakly imposed boundary conditions on 100 cubic B-spline elements with element size $h_{e}=0.1$. Figure 3 plots the corresponding concentration $c$ and the free energy $\psi$ at equilibrium $\left(t \sim 10^{13}\right)$, the latter being computed as

$$
\psi=\psi_{c}+\frac{1}{2} \kappa|\nabla c|^{2}=c \ln c+(1-c) \ln (1-c)+2.5 c(1-c)+5 \times 10^{-3}\left|\frac{\partial c}{\partial x}\right|^{2} .
$$

There are two phases and a diffuse interface, with the energetic peak in the center, where the largest concentration gradient occurs. Performing a simple analytical study, we find the exact free energy at $\psi=-0.104$ in the homogeneous phases, with a peak value of $\psi=-0.0323$ when $c=0.5$. We observe in Figure 3 that the analytical results are accurately reproduced by the simulation.

In the next step, we perform a more rigorous convergence study. We know that at equilibrium, the exact chemical potential $\mu_{\mathrm{ex}}=0$ holds everywhere in the field, while there is no analytical 


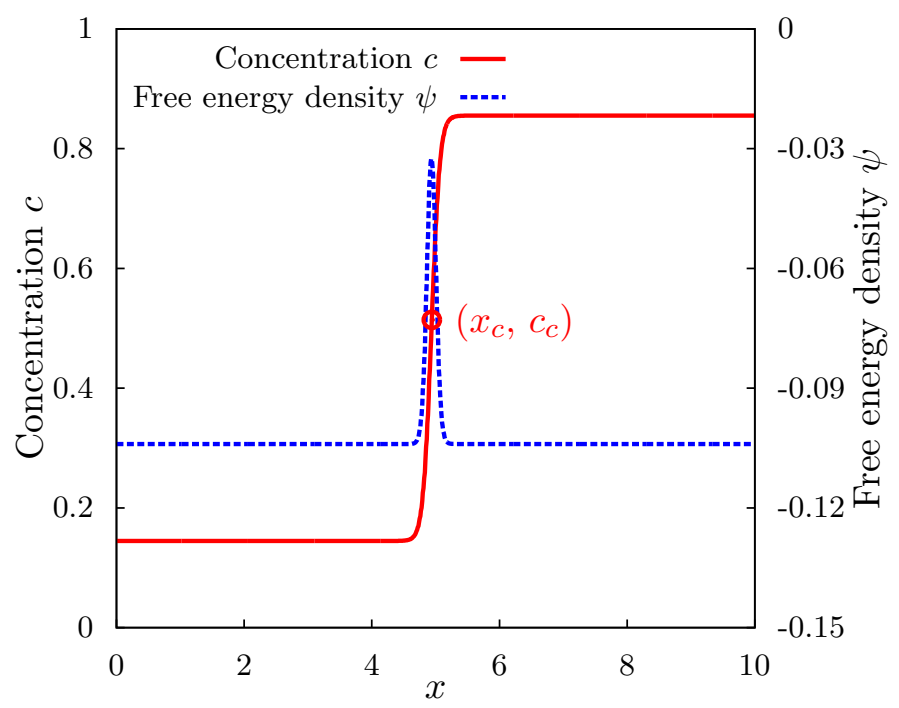

FIGURE 3: The numerical solution of the concentration $c$ and the free energy $\psi$ at equilibrium. The analytical solution for the two phases are $c_{\alpha}=0.1448$ and $c_{\beta}=0.8552$. The free energy is $\psi=-0.1040$ at homogeneous phases and the peak value is $\psi=-0.03229$ when $c=c_{c}=0.5$ in the middle of the interface. All numerical results agree well with the analytical results.

solution for $c$. We therefore base our study on the chemical potential

$$
\mu=\ln c-\ln (1-c)+2.5(1-2 c)-1 \times 10^{-2} \frac{\partial^{2} c}{\partial x^{2}}
$$

instead of the concentration $c$. Since this example does not involve a Dirichlet boundary condition, we only use the stabilization parameter $\alpha_{2}$. Following the work of Wells et al. [5], the penalty parameter $\alpha_{2}$ is taken as $5 / h_{e}$, where $h_{e}$ is the characteristic length of each boundary element.

Table 1 and Figure 4 show the convergence of the error in the $\mathrm{L}_{2}$ norm, computed for $\mu$. We observe that for quadratic and quartic B-splines, we achieve the optimal convergence rate of two and four, respectively. For cubic basis functions, we achieve a superconvergent rate of four. This can be explained as follows: Inspection of the free energy $\psi(28)$ indicates that the distribution of $c$ follows a sigmoid shape, which possesses rotational symmetry with respect to the middle point of the interface $\left(x_{c}, c_{c}\right)$ (see Figure 3) [1]. This means that in the vicinity of $\left(x_{c}, c_{c}\right)$, only odd power terms are non-zero in a Taylor expansion of $c$

$$
c(x)=c\left(x_{c}\right)+\sum_{n=1,3, \ldots} A_{n}\left(x-x_{c}\right)^{n}=c_{c}+\sum_{n=1,3, \ldots} A_{n}\left(x-x_{c}\right)^{n},
$$

with $A_{n}$ being the coefficients of the $n$-th term. Since $\mu$ is a function of $c$ and $\mathrm{d}^{2} c / \mathrm{d} x^{2}$, the monomial components of the polynomial basis functions that have even power must have coefficients of zero and therefore do not contribute to the accuracy for $\mu$ at equilibrium state. Therefore, odd degree cubics achieve the same accuracy as quartics in this special case. 


\begin{tabular}{llll}
\hline $1 / h_{e}$ & Quadratics & Cubics & Quartics \\
\hline 10 & $7.3845721536 \times 10^{-3}$ & $1.5582188728 \times 10^{-3}$ & $3.7061461505 \times 10^{-4}$ \\
20 & $1.4062186408 \times 10^{-3}$ & $6.2066527184 \times 10^{-5}$ & $1.7353482112 \times 10^{-5}$ \\
40 & $2.5452237937 \times 10^{-4}$ & $2.5201024011 \times 10^{-6}$ & $6.3594095903 \times 10^{-7}$ \\
80 & $4.5261342896 \times 10^{-5}$ & $1.0874341541 \times 10^{-7}$ & $2.6685542218 \times 10^{-8}$ \\
160 & $8.0130442296 \times 10^{-6}$ & $4.7848784864 \times 10^{-9}$ & $1.2567716120 \times 10^{-9}$ \\
\hline
\end{tabular}

TABLE 1: Convergence of $\left[\int_{0}^{L}\left(\mu_{\mathrm{FEM}}-\mu_{\mathrm{ex}}\right)^{2} \mathrm{~d} x\right]^{\frac{1}{2}}$.

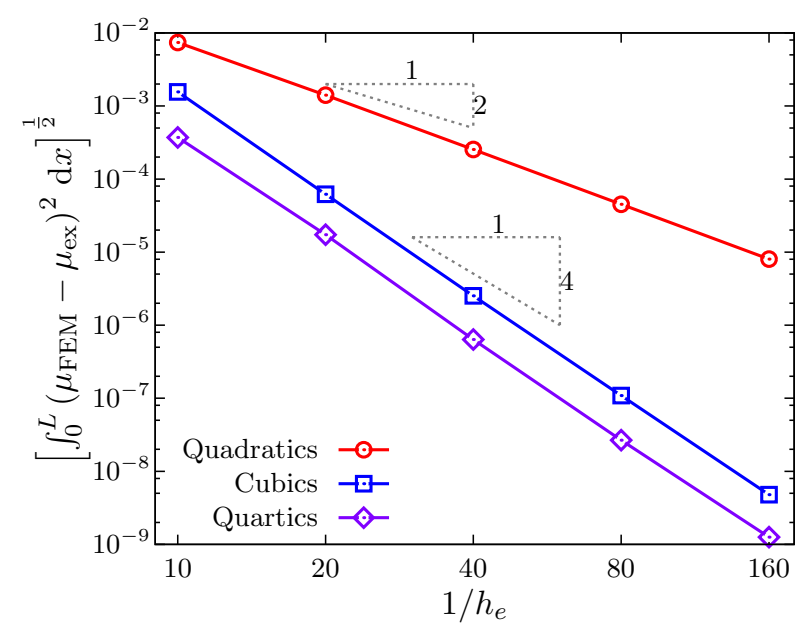

FIGURE 4: Plot of the convergence of $\left[\int_{0}^{L}\left(\mu_{\mathrm{FEM}}-\mu_{\mathrm{ex}}\right)^{2} \mathrm{~d} x\right]^{\frac{1}{2}}$.

We also consider a second scenario, where one of the flux boundary conditions is replaced by a corresponding Dirichlet boundary condition. The new set reads

$$
\begin{array}{ll}
c=1.0, & x=0, \quad t \in(0, \mathrm{~T}), \\
{[1-2 \chi c(1-c)] \frac{\partial c}{\partial x}-c(1-c) \kappa \frac{\partial^{3} c}{\partial x^{3}}=0,} & x=\mathrm{L}, \quad t \in(0, \mathrm{~T}), \\
\kappa \frac{\partial c}{\partial x}=0, & x=0, \mathrm{~L}, \quad t \in(0, \mathrm{~T}) .
\end{array}
$$

The initial condition is the homogeneous concentration

$$
c(x, 0)=0.1, \quad x \in[0, \mathrm{~L}] .
$$

We enforce both conditions (31) and (33) weakly, which involves two stabilization terms. Following Embar et al. [24], we assume a stabilization parameter $\alpha_{1}=5000 / h_{e}$, that is three orders of magnitude larger than the second stabilization parameter $\alpha_{2}$.

Since the fixed concentration $c=1.0$ at $x=0$ is larger than the equilibrium concentrations 


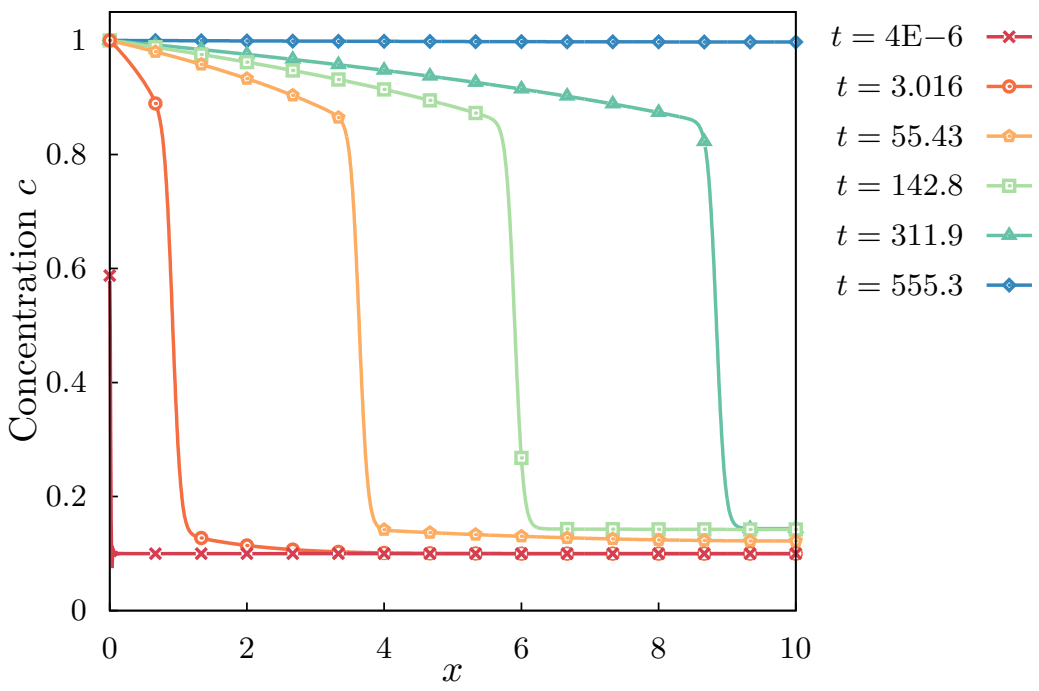

FIGURE 5: Evolution of the concentration profile with a Dirichlet boundary condition at $x=0$ and zero first derivatives at both ends, all weakly enforced.

$c_{\alpha}=0.1448$ and $c_{\beta}=0.8552$, it triggers a continuous influx until the overall domain reaches a homogeneous concentration of $c=1.0$. Figure 5 shows the concentration evolution in the bar, where we use 500 cubic B-spline elements, $\alpha_{1}=2.5 \times 10^{5}$ and $\alpha_{2}=250$. We observe that the Dirichlet boundary condition is accurately satisfied at all times.

\subsection{Spinodal decomposition in a $2 D$ unit square domain}

In the second example, we consider spinodal decomposition on a unit square from a random distributed initial concentration field. We assume parameters $\chi=2.5$ and $\kappa=1.42 \times 10^{-4}$, which leads to a characteristic interfacial thickness of 0.0316 . The initial concentration is

$$
c(\boldsymbol{x}, 0)=\bar{c}+r
$$

where $\bar{c}=0.63$ and $r$ is a random variable with uniform distribution in the range $[-0.05,0.05]$.

In a first step, we examine the evolution under a natural boundary condition. In this case, the flux on all boundaries and the normal gradient of the concentration are zero, the latter being enforced weakly by the Nitsche method. Figure 6 shows the evolution of the concentration field at different times, computed with $128 \times 128$ cubic B-spline elements. It is observed that phase interfaces are always perpendicular to the boundary when they intersect with the boundary. This indicates that the weakly enforced condition of zero normal gradient is accurately satisfied on the complete boundary.

In a second step, we simulate the evolution of the random field under weakly enforced periodic boundary conditions (19)-(21). The constraint of Eq. (18) is imposed strongly by making the corresponding two control points on the edge coincide. We assume $\alpha_{2}=5.0 / h_{e}$. Figure 7 plots simulation results at different time instances, computed again with $128 \times 128$ cubic B-spline elements. We observe that phase interfaces now continue smoothly from one boundary to the 
opposite boundary. The final equilibrium state is a complete circle. This is different from the previous case, where only one quarter of the complete sphere is reached at the equilibrium, and where it is energetically more favorable. This can be observed by computing the final energy. For both simulations, a monotonic decrease of the total free energy

$$
\Psi=\int_{\Omega} \psi \mathrm{d} \Omega=\int_{\Omega}\left[c \ln c+(1-c) \ln (1-c)+2.5 c(1-c)+7.1 \times 10^{-5}|\nabla c|^{2}\right] \mathrm{d} \Omega
$$

with time is observed. Moreover, the final total energy of the species under natural boundary conditions (Figure 8) is lower than that under periodic boundary conditions (Figure 9).

\subsection{Spheroidal particles with incoming flux}

As the third example, a $3 \mathrm{D}$ prolate spheroid with the aspect ratio $3 / 2: \sqrt{2 / 3}: \sqrt{2 / 3}$ is considered. Due to symmetry, only an octant of the whole spheroid is simulated. Homogeneous flux is applied on the surface for 2 normalized seconds and removed afterwards. After removal of the flux, the species in the particle evolve towards the equilibrium. The simulation parameters are listed in Table 2, For the simulation, quadratic NURBS shape functions are used. The mesh

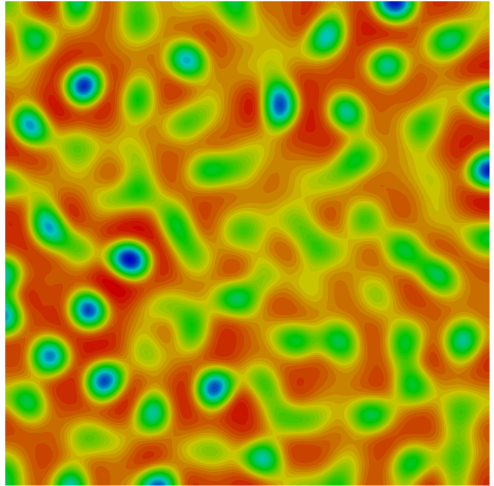

(a) $t=9.83 \times 10^{-3}$

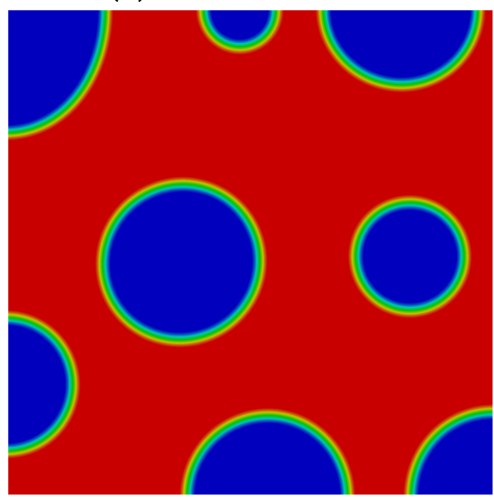

(d) $t=5.57$

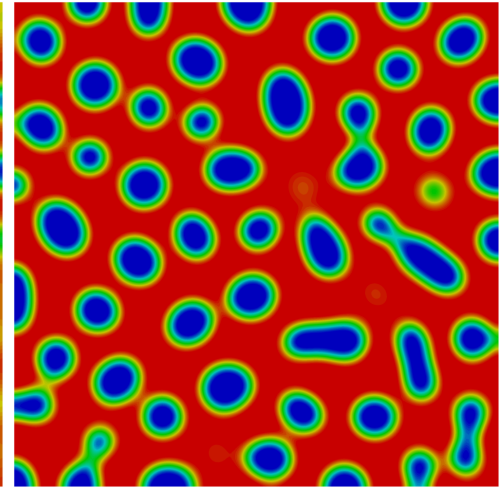

(b) $t=2.576 \times 10^{-2}$

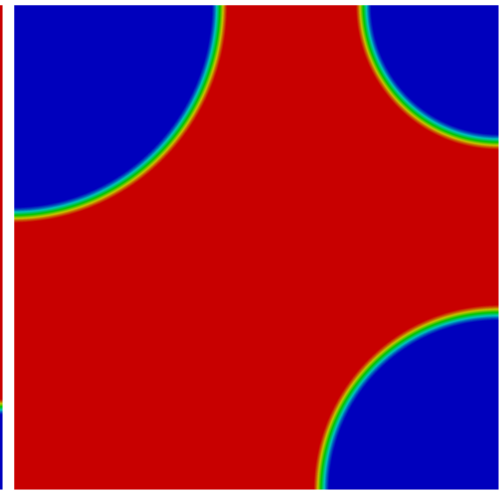

(e) $t=62.01$

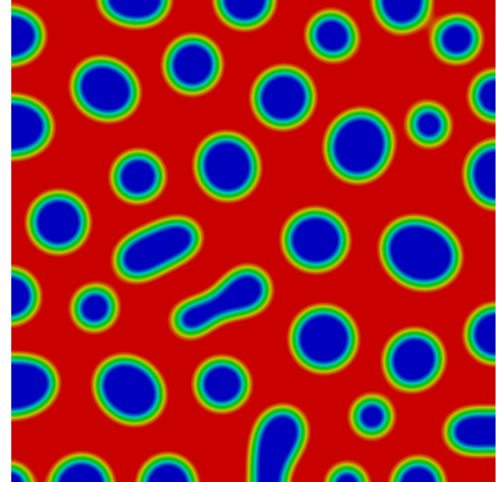

(c) $t=1.056 \times 10^{-1}$

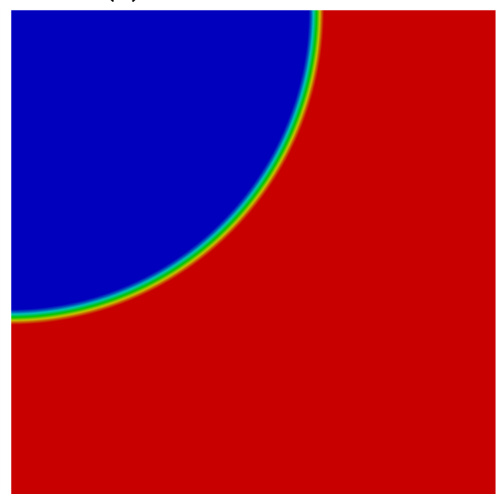

(f) Equilibrium

FIGURE 6: Spinodal decomposition with natural boundary conditions. Due to the weakly enforced condition of zero normal gradient, all phase interfaces remain perpendicular to the boundary. 


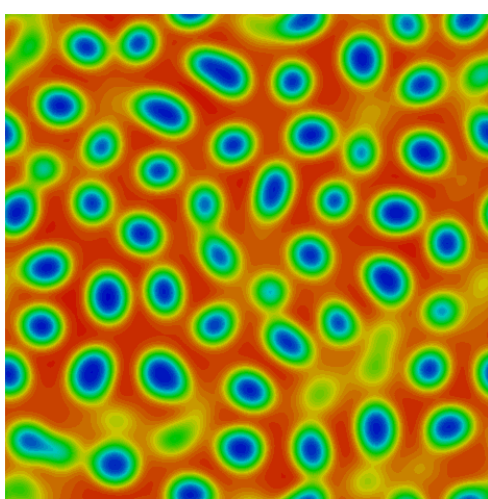

(a) $t=1.581 \times 10^{-2}$

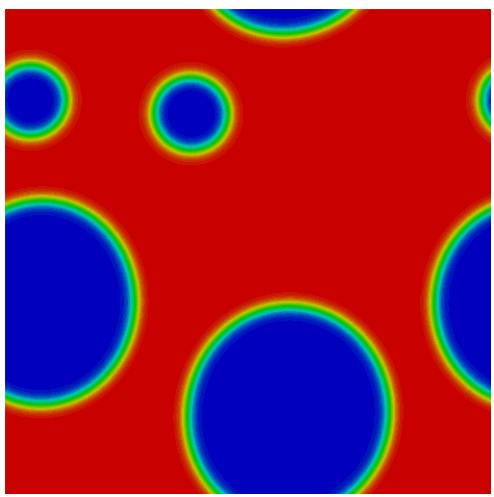

(d) $t=10.28$

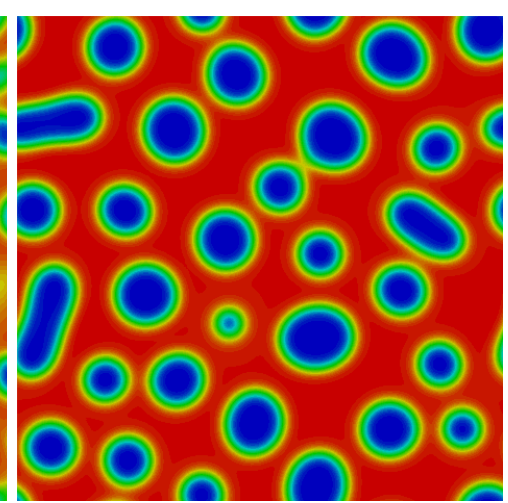

(b) $t=1.153 \times 10^{-1}$

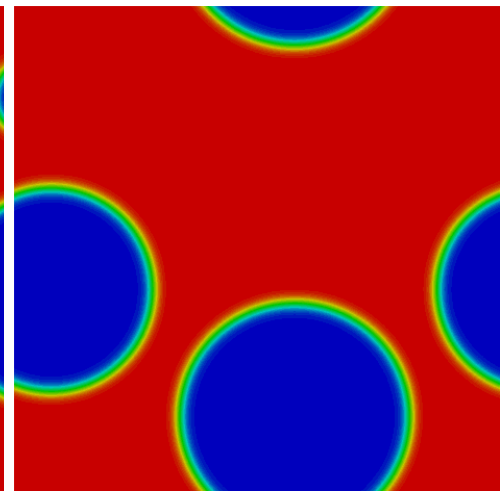

(e) $t=20.41$

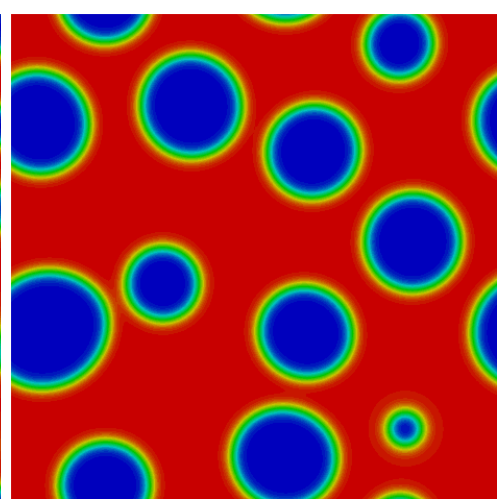

(c) $t=1.231$

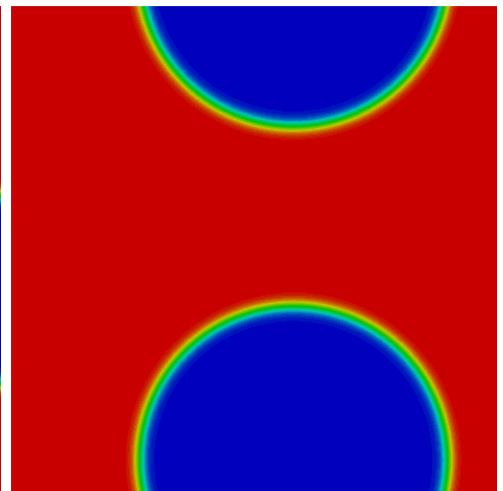

(f) Equilibrium

FIGURE 7: Spinodal decomposition under periodic boundary conditions. $128^{2}$ cubic quadrilateral elements are used in this simulation. The phase interfaces are not perpendicular but continue to the opposite side of the border when intersected with the box frame.

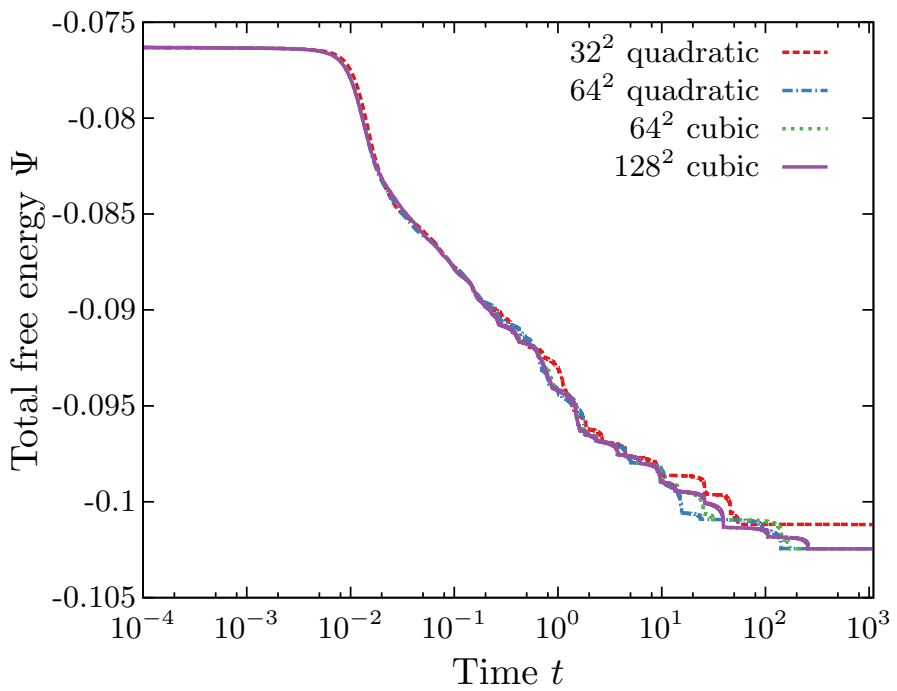

FIGURE 8: Evolution of the total free energy $\Psi$ with different meshes under natural boundary conditions. 


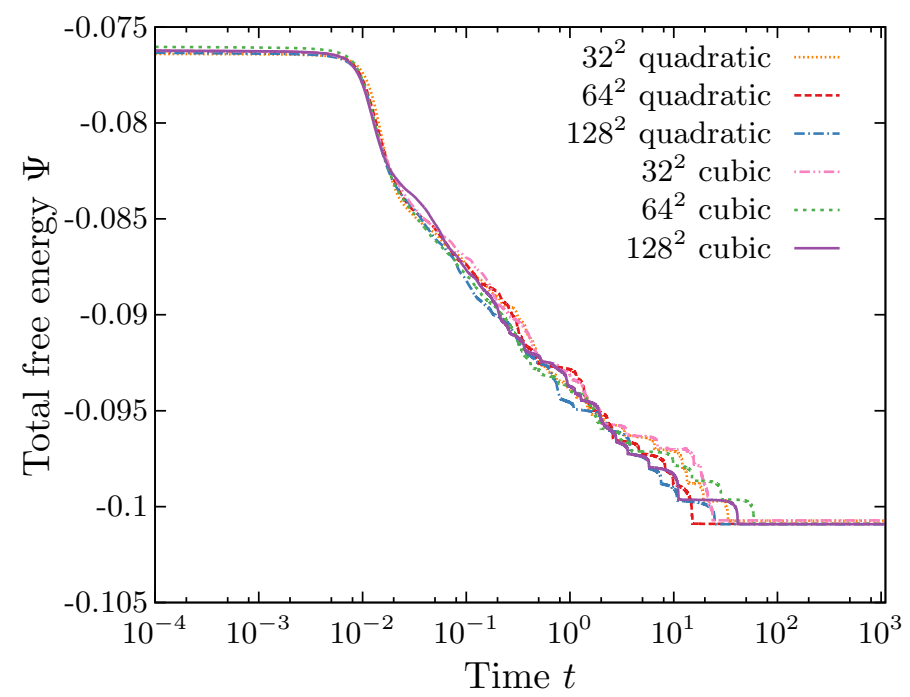

FIGURE 9: Evolution of the total free energy $\Psi$ with different meshes under periodic boundary conditions.

\begin{tabular}{rc}
\hline Phase parameter $\chi$ & 2.5 \\
Interface parameter $\kappa$ & $4.25 \times 10^{-3}$ \\
Flux per area $\hat{j}$ & -0.1 \\
Time flux applied $\mathrm{T}_{\text {app }}$ & 2 \\
Initial concentration $c_{0}$ & 0.25 \\
\hline
\end{tabular}

TABLE 2: Simulation parameters for the 3D spheroids

and the control points are plotted in Figure 10 . It is obvious from the figure that radial control lines are not perpendicular to the surface, which makes it impossible to impose the boundary condition of $\nabla c \cdot \boldsymbol{n}=0$ strongly as described in [10, 14]. For more details about the mesh readers are referred to Zhao et al. [11] and Stein et al. [49]. With the examples in this section, we would like to demonstrate the ability to constrain the boundary condition $\boldsymbol{\nabla} c \cdot \boldsymbol{n}=0$, when the boundary is perturbed by an incoming flux. Figure 11 shows the simulation results at different time instants. As a comparison, the results with the method described in Zhao et al. [11] is also plotted, where a Lagrange multiplier $\lambda$ is introduced for the additional constraint. The weak formulation reads

$$
\begin{aligned}
& \int_{\Omega} \frac{\partial c}{\partial t} \delta c \mathrm{~d} \Omega+\int_{\Omega}[1-2 \chi c(1-c)] \nabla c \cdot \nabla \delta c \mathrm{~d} \Omega+\int_{\Omega}(1-2 c) \kappa \Delta c \boldsymbol{\nabla} c \cdot \nabla \delta c \mathrm{~d} \Omega \\
& +\int_{\Omega} c(1-c) \kappa \Delta c \Delta \delta c \mathrm{~d} \Omega+\delta \int_{\Omega} \operatorname{Div}(\lambda \nabla c) \mathrm{d} \Omega-\delta \int_{\Omega} \frac{1}{2 \alpha} \lambda^{2} \mathrm{~d} \Omega=\int_{\partial \Omega} j \delta c \mathrm{~d} \Gamma
\end{aligned}
$$

The stabilization parameter $\alpha$ in the simulation is chosen empirically as $10^{2}$. For a better comparison, a fixed time step 0.01 is used here. It is shown that the two methods give the same evolution of the concentration field. A core-shell two-phase structure is maintained when the flux comes in. The phase interface initiates on the surface and marches stably towards the center until the 


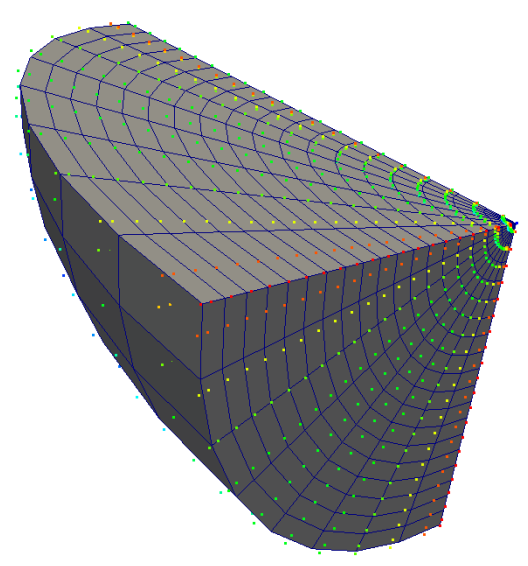

(a) The 3D mesh.

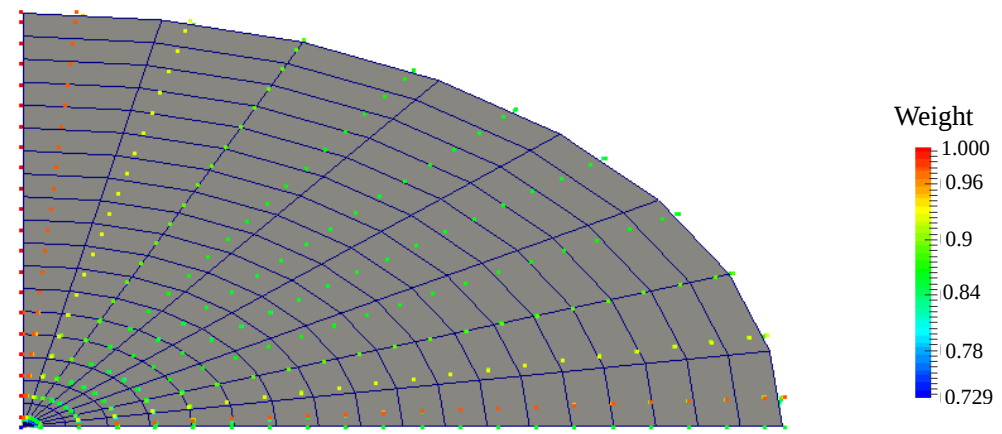

(b) The mesh and control points of one plane of symmetry.

FIGURE 10: The mesh and the control points of the prolate spheroid. The radial control lines are not perpendicular to the surface. A strong imposition of $\nabla c \cdot \boldsymbol{n}=0$ is not possible. The weight of each control point is indicated by the color.

total amount of flux is large enough to suppress the phase segregation. The phase interfaces are not spherical due to the geometry and the flux. To demonstrate that the constraint $\boldsymbol{\nabla} c \cdot \boldsymbol{n}=0$ is accurately satisfied, we plot the distribution of its residual on the spheroidal surface in Figure 12 . The evolution of $\boldsymbol{\nabla} c \cdot \boldsymbol{n}$ with time at a representative point P (shown in Figure 12a) is plotted in Figure 12c From the plots one can observe that both methods show similar behavior for the constraint, while the Lagrange multiplier method gives stronger oscillations of $\boldsymbol{\nabla} c \cdot \boldsymbol{n}$, especially when the surface is strongly perturbed by the incoming flux.

\section{Simulation of chemical phase segregation in composite electrodes on unfitted Carte- sian B-spline meshes}

In composite electrodes of lithium-ion batteries, particles made of active materials, such as silicon, are embedded in a matrix. The matrix usually consists of polymer binders and conductive additives, which are electrochemically inactive, and offer the mechanical and electronic support to the active particles in the composite electrodes [50-52]. Their amount of content plays a crucial rule in the performance of lithium-ion batteries [53]. However, the study on the interaction between the particles and the binders is still far less than that on the active electrode particles. Doyle et al. [54] presented a cathode cell, which included a wide range of polymeric separator materials, lithium salts, and composite insertion cathodes. Bower et al. [55] formulated a continuum electro-chemo-mechanical half-cell model in the large deformation framework with plastic flow. Furthermore, there are multi-scale approaches of lithium-ion battery cells, where the particles embedded in the polymer binders are modeled at the micro-scale [56, 57]. For a fair representation of geometric irregularity, Lee et al. [57] generated the microstructure randomly. As a more faithful attempt, Roberts et al. [58] and Mendoza et al. [59] employed the Conformal Decomposition Finite Element Method introduced by Noble et al. [60] for a reconstruction of the microstructure. Orvananos et al. [61] considered the interaction between electrode nanoparticles 


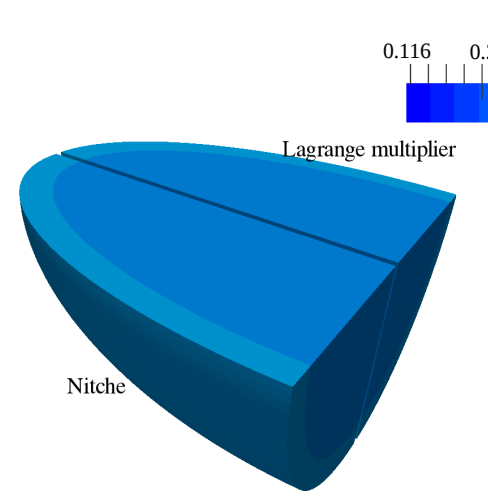

(a) $t=0.01$

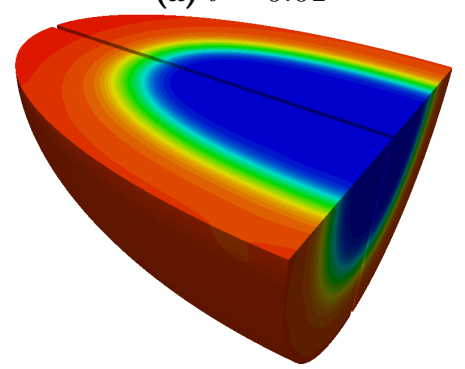

(d) $t=1.30$

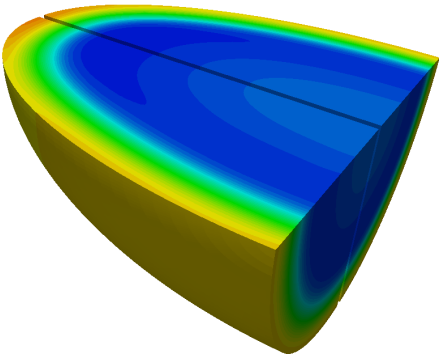

(b) $t=0.44$

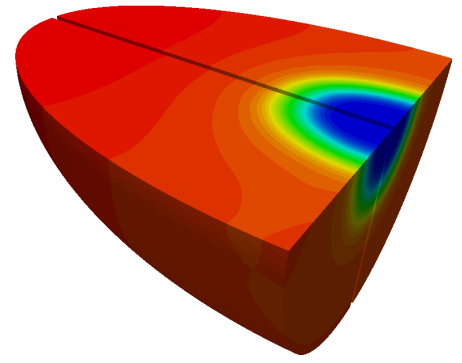

(e) $t=2.20$

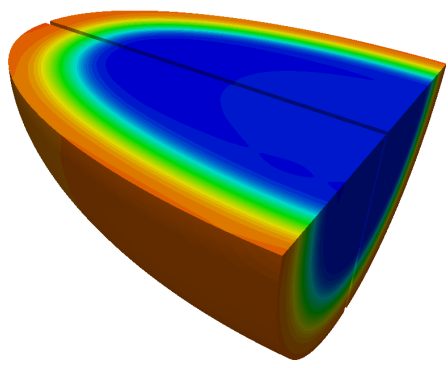

(c) $t=0.68$

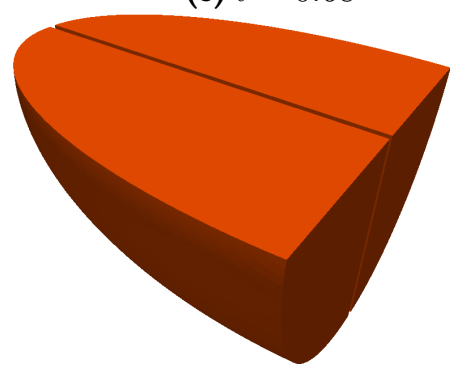

(f) $t=3.98$

FIGURE 11: Evolution of the concentration in a prolate spheroid with an incoming flux. The two octants are computed with different methods: the lower left is with the Nitsche method, while the upper right octant is computed by the method from [11] with a Lagrange multiplier. 


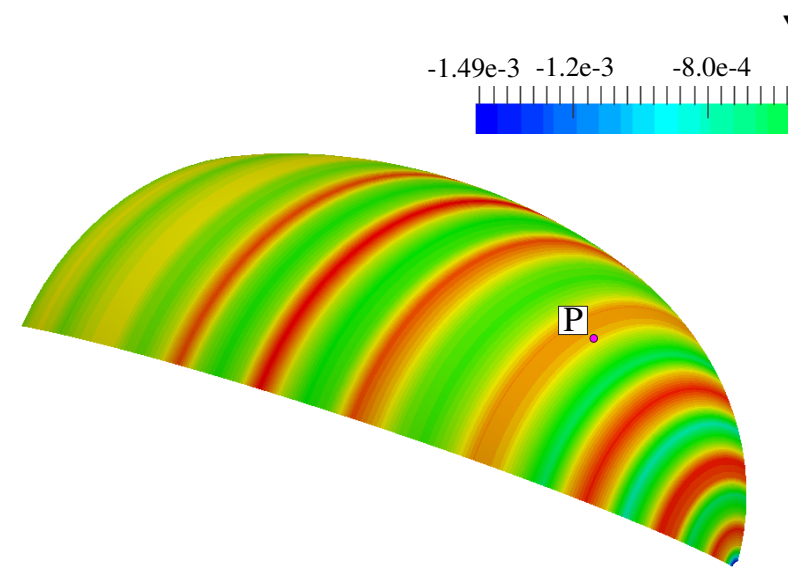

(a) Nitsche $\boldsymbol{\nabla} c \cdot \boldsymbol{n}$

$\begin{array}{llll}-4.0 \mathrm{e}-4 & 0.0 \quad 4.0 \mathrm{e}-4 & 6.49 \mathrm{e}-4\end{array}$
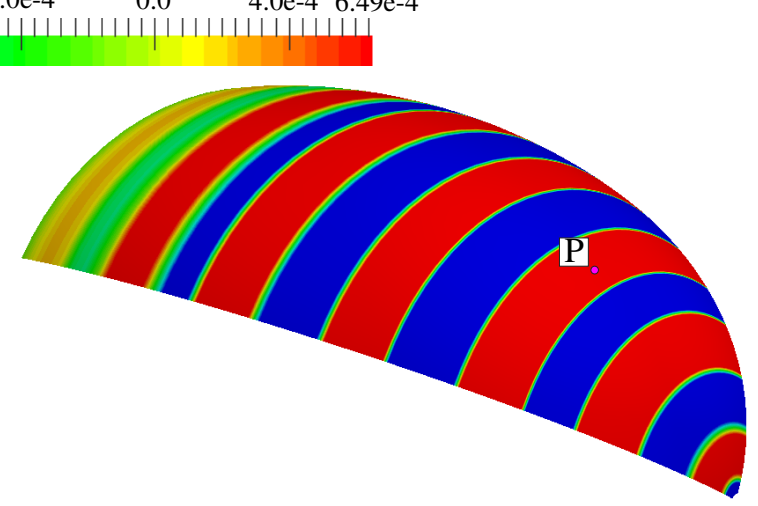

(b) Lagrange multiplier

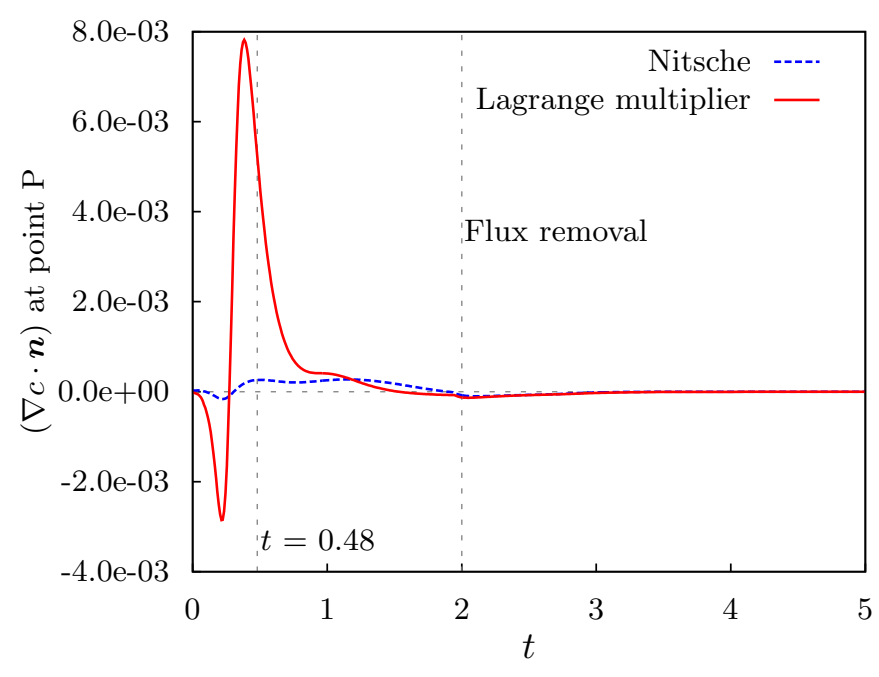

(c) Evolution of $\nabla c \cdot \boldsymbol{n}$ at Point $P$.

FIGURE 12: Distribution of the residual of $\nabla c \cdot n$ on the surface at $t=0.48$ with the Nitsche method (a) and Lagrange multiplier method (b). Both show a similar pattern, while the Nitsche method gives a better constraint than the Lagrange multiplier method at that time. For a quantitative comparison, the evolution of $\nabla c \cdot n$ at a representative point $P$ (shown in (a) and (b)) is plotted in (c). The Lagrange multiplier method gives a stronger oscillation when the surface is perturbed by the incoming flux, but both methods bring $\boldsymbol{\nabla} c \cdot \boldsymbol{n}$ to zero once the flux is removed. 


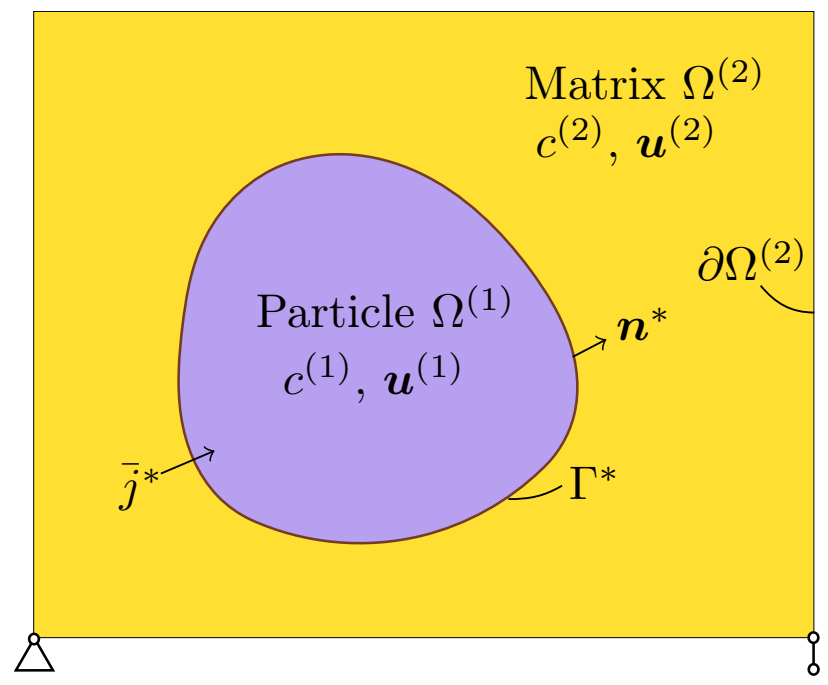

FIGURE 13: Composite electrode configuration: The particle is constrained by a simply supported matrix. Different conditions are prescribed weakly at the interface $\Gamma^{*}$ and the matrix boundary $\partial \Omega^{(2)}$.

and the interface condition between the particle, where the electrolyte is modeled by a smoothed boundary method.

In this section, we present the chemo-mechanical modeling of the composite electrode with phase segregation and perform unfitted finite element simulations with the finite cell method. This allows for a flexible representation of irregular geometries.

\subsection{Governing equations of the composite electrode}

We consider the one-particle configuration shown in Fugure 13 . The particle, defined on domain $\Omega^{(1)}$, is embedded in the matrix that occupies $\Omega^{(2)}$. The interface between the two domains is denoted by $\Gamma^{*}=\Omega^{(1)} \cap \Omega^{(2)}$. In each domain, there are two coupled fields: the displacement field $\boldsymbol{u}^{(p)}$ and the concentration field $c^{(p)}$. Mechanically, the matrix and the particle are linear elastic. Displacements and normal traction across the interface are continuous.

In each domain, there are two sets of equations that govern the mechanical and the chemical behavior, respectively. We note that in this section, the domain index $p$ runs from 1 to 2 . For the mechanical part, the local force balance states in each domain $\Omega^{(p)}$

$$
\boldsymbol{\nabla} \cdot \boldsymbol{\sigma}^{(p)}=\boldsymbol{\nabla} \cdot\left(\mathbb{C}^{(p)} \boldsymbol{\epsilon}^{(p)}\right)-\mathrm{K}^{(p)} \mathrm{V}^{(p)} \boldsymbol{\nabla} c^{(p)}=\mathbf{0},
$$

The fourth-order elastic tensor $\mathbb{C}^{(p)}$ is given as

$$
\mathbb{C}^{(p)}=2 \mathrm{G}^{(p)} \mathbb{I}+\left(\mathrm{K}^{(p)}-\frac{2}{3} \mathrm{G}^{(p)}\right) \mathbf{1} \otimes \mathbf{1},
$$

with $\mathrm{G}^{(p)}$ and $\mathrm{K}^{(p)}$ being the shear and bulk modulus, respectively. I is the fourth-order and $\mathbf{1}$ is the second-order unit tensor. The strains $\epsilon^{(p)}$ are the symmetric part of the displacement gradient 


$$
\boldsymbol{\epsilon}^{(p)}=\frac{1}{2}\left(\boldsymbol{\nabla} \boldsymbol{u}^{(p)}+\left(\boldsymbol{\nabla} \boldsymbol{u}^{(p)}\right)^{\mathrm{T}}\right) .
$$
with proportionality factor as the partial molar volume $\mathrm{V}^{(p)}$.

Chemically, lithium cations diffuse from the matrix towards the electrode particle. While the particle experiences phase segregation upon the incoming flux and the Cahn-Hilliard equation applies, the diffusion in the matrix follows the stress assisted Fick law. Therefore, since there exist phase segregation, inside the particle, the diffusion process is governed by the stress-assisted Cahn-Hilliard equation

$$
\begin{aligned}
\frac{\partial c^{(1)}}{\partial t} & =\boldsymbol{\nabla} \cdot\left[c^{(1)}\left(1-c^{(1)}\right) \boldsymbol{\nabla} \mu^{(1)}\right] \\
& =\boldsymbol{\nabla} \cdot\left\{\left[1-2 \chi^{(1)} c^{(1)}\left(1-c^{(1)}\right)\right] \boldsymbol{\nabla} c^{(1)}-c^{(1)}\left(1-c^{(1)}\right) \kappa^{(1)} \boldsymbol{\nabla} \Delta c^{(1)}\right\} \\
& -\boldsymbol{\nabla} \cdot\left[c^{(1)}\left(1-c^{(1)}\right) \frac{1}{3} \mathrm{~V}^{(1)} \boldsymbol{\nabla}\left(\operatorname{tr} \boldsymbol{\sigma}^{(1)}\right)\right] \quad \text { in } \quad \Omega^{(1)} \times(0, \mathrm{~T}) .
\end{aligned}
$$

In the matrix, one-phase diffusion takes place as

$$
\begin{aligned}
\frac{\partial c^{(2)}}{\partial t} & =\boldsymbol{\nabla} \cdot\left[c^{(2)}\left(1-c^{(2)}\right) \boldsymbol{\nabla} \mu^{(2)}\right] \\
& =\boldsymbol{\nabla} \cdot\left\{\left[1-2 \chi^{(2)} c^{(2)}\left(1-c^{(2)}\right)\right] \boldsymbol{\nabla} c^{(2)}-c^{(2)}\left(1-c^{(2)}\right) \frac{1}{3} \mathrm{~V}^{(2)} \boldsymbol{\nabla}\left(\operatorname{tr} \boldsymbol{\sigma}^{(2)}\right)\right\} \\
& \text { in } \quad \Omega^{(2)} \times(0, \mathrm{~T}) .
\end{aligned}
$$

The parameters $\chi^{(1)}, \chi^{(2)}$ and $\kappa^{(1)}$ in (41) and (42) have been already introduced in Section 2.1 The initial condition in the two materials are

$$
\begin{array}{lll}
c^{(1)}=c_{0}^{(1)} & \text { in } & \Omega^{(1)} \\
c^{(2)}=c_{0}^{(2)} & \text { in } & \Omega^{(2)}
\end{array}
$$

For more details on the material model, the readers are referred to our previous work [11, 12, 62].

On the interface between two domains $\Gamma^{*}$, the mechanical compatibility conditions are

$$
\begin{aligned}
& \boldsymbol{u}^{(1)}-\boldsymbol{u}^{(2)}=\llbracket \boldsymbol{u} \rrbracket=\mathbf{0}, \\
& \boldsymbol{\sigma}^{(1)} \boldsymbol{n}^{*}-\boldsymbol{\sigma}^{(2)} \boldsymbol{n}^{*}=\llbracket \boldsymbol{\sigma} \rrbracket=\mathbf{0},
\end{aligned}
$$

with $\boldsymbol{n}^{*}$ being the normal vector of $\Gamma^{*}$. For the chemical part, mass conservation across the interface is expressed as

$$
\begin{array}{ll}
-\left[c^{(1)}\left(1-c^{(1)}\right) \nabla \mu^{(1)}\right] \cdot \boldsymbol{n}^{*}=-\bar{j}^{*} & \text { on } \quad \Gamma^{*} \times(0, \mathrm{~T}), \\
-\left[c^{(2)}\left(1-c^{(2)}\right) \nabla \mu^{(2)}\right] \cdot \boldsymbol{n}^{*}=-\bar{j}^{*} & \text { on } \quad \Gamma^{*} \times(0, \mathrm{~T}),
\end{array}
$$




$$
\kappa^{(1)} \nabla c^{(1)} \cdot \boldsymbol{n}^{*}=0 \quad \text { on } \quad \Gamma^{*} \times(0, \mathrm{~T}),
$$

where $\bar{j}^{*}$ is determined by the reaction rate, which in this paper is assumed constant. The additional condition (49) arises due to the Cahn-Hilliard equation (41) as discussed in Section 2.2

The boundary conditions on the surface $\partial \Omega^{(2)}$ consist of two parts. Mechanically, only minimum constraints are applied to the matrix in order to prevent rigid body movement (see Figure 13. Chemically, Dirichlet boundary conditions are prescribed as

$$
c^{(2)}=\bar{c} \quad \text { on } \quad \partial \Omega^{(2)} \times(0, \mathrm{~T}) .
$$

\subsection{The Nitsche method for the chemo-mechanically coupled multi-field problem}

The weak formulation of the problem described in Section 5.1 is

$$
\begin{aligned}
& 0=\sum_{p=1,2} \int_{\Omega^{(p)}} \frac{\partial c^{(p)}}{\partial t} \delta c^{(p)} \mathrm{d} \Omega+\sum_{p=1,2} \int_{\Omega^{(p)}}\left[1-2 \chi^{(p)} c^{(p)}\left(1-c^{(p)}\right)\right] \nabla c^{(p)} \cdot \nabla \delta c^{(p)} \mathrm{d} \Omega \\
& -\sum_{p=1,2} \int_{\Omega^{(p)}} \frac{1}{3} \mathrm{~V}^{(p)} c^{(p)}\left(1-c^{(p)}\right) \boldsymbol{\nabla}\left(\operatorname{tr} \boldsymbol{\sigma}^{(p)}\right) \cdot \nabla \delta c^{(p)} \mathrm{d} \Omega \\
& +\int_{\Omega^{(1)}}\left(1-2 c^{(1)}\right) \kappa^{(1)} \Delta c^{(1)} \nabla c^{(1)} \cdot \nabla \delta c^{(1)} \mathrm{d} \Omega \\
& +\int_{\Omega^{(1)}} c^{(1)}\left(1-c^{(1)}\right) \kappa^{(1)} \Delta c^{(1)} \Delta \delta c^{(1)} \mathrm{d} \Omega-\sum_{p=1,2} \int_{\Omega^{(p)}} \boldsymbol{\sigma}^{(p)}: \delta \boldsymbol{\epsilon}^{(p)} \mathrm{d} \Omega \\
& -\int_{\Gamma^{*}} \bar{j}^{*} \delta c^{(1)} \mathrm{d} \Gamma+\int_{\Gamma^{*}} \bar{j}^{*} \delta c^{(2)} \mathrm{d} \Gamma+\int_{\Gamma^{*}}\langle\boldsymbol{\sigma}\rangle \cdot \llbracket \delta \boldsymbol{u} \rrbracket \mathrm{d} \Gamma-\int_{\Gamma^{*}} \llbracket \boldsymbol{u} \rrbracket \cdot\langle\delta \boldsymbol{\sigma}\rangle \mathrm{d} \Gamma \\
& -\int_{\Gamma^{*}} c^{(1)}\left(1-c^{(1)}\right) \kappa^{(1)} \Delta c^{(1)} \boldsymbol{n}^{*} \cdot \nabla \delta c^{(1)} \mathrm{d} \Gamma \\
& -\int_{\Gamma^{*}} c^{(1)}\left(1-c^{(1)}\right) \boldsymbol{\nabla} c^{(1)} \cdot \boldsymbol{n}^{*} \kappa^{(1)} \Delta \delta c^{(1)} \mathrm{d} \Gamma+\alpha_{2} \int_{\Gamma^{*}}\left(\boldsymbol{\nabla} c^{(1)} \cdot \boldsymbol{n}^{*}\right)\left(\boldsymbol{\nabla} \delta c^{(1)} \cdot \boldsymbol{n}^{*}\right) \mathrm{d} \Gamma .
\end{aligned}
$$

The additional mechanical coupling conditions Eq. (45) and 46) are imposed by the non-symmetric Nitsche method [63] with the form

$$
0=-\sum_{p=1,2} \int_{\Omega^{(p)}} \boldsymbol{\sigma}^{(p)}: \delta \boldsymbol{\epsilon}^{(p)} \mathrm{d} \Omega+\int_{\Gamma^{*}}\langle\boldsymbol{\sigma}\rangle \cdot \llbracket \delta \boldsymbol{u} \rrbracket \mathrm{d} \Gamma-\int_{\Gamma^{*}} \llbracket \boldsymbol{u} \rrbracket \cdot\langle\delta \boldsymbol{\sigma}\rangle \mathrm{d} \Gamma,
$$

which offers a parameter-free way of weakly enforcing interface constraints. Moreover, its nonsymmetrix contributions do not affect the symmetry of the stiffness matrix, since in the chemomechanically coupled problem, the stiffness matrix is already non-symmetric.

\subsection{The phase segregation inside the particle}

As the first example, we consider a standalone particle, where we also disregard the mechanical stresses. In this way, the problem degenerates to a pure Cahn-Hilliard equation in the particle. This enables us to monitor the performance of the finite cell method and to compare it with corresponding results obtained by isogeometric analysis with an exact geometric description. Figure 14 illustrates the numerical integration of intersected elements in the finite cell method. 


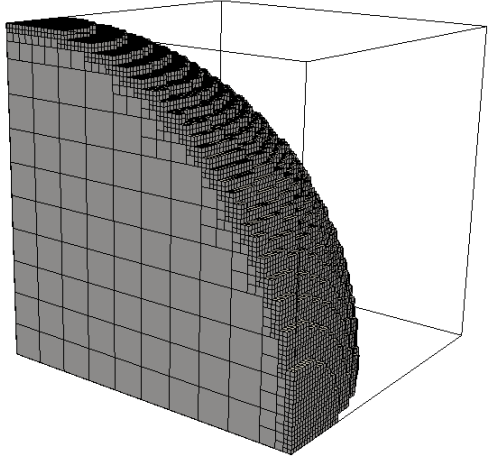

(a) Sub-cells

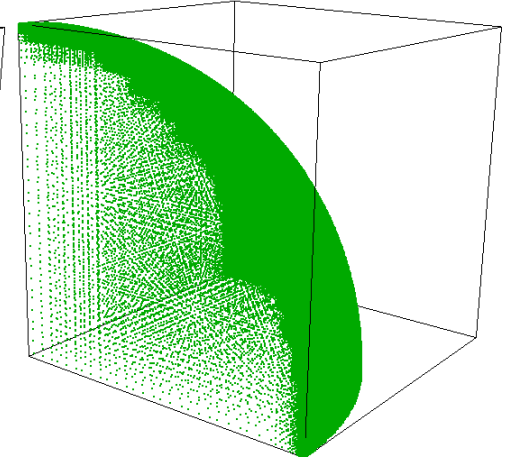

(b) Volume quadratures

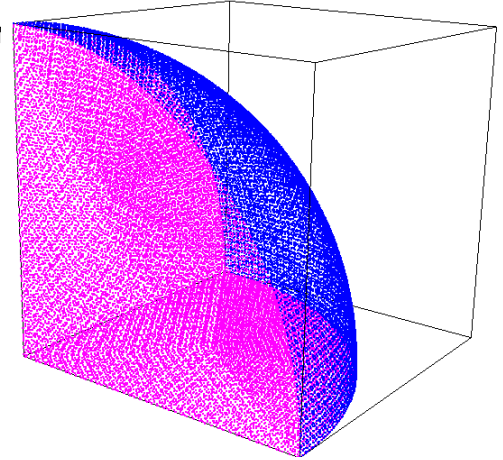

(c) Surface quadratures

FIGURE 14: Numerical integration of intersected elements in the finite cell method. The volume quadrature points in (b) are computed based on adaptive subdivision to the third level of the original $10^{3}$ Cartesian elements (a). The boundary condition of $\boldsymbol{\nabla} c \cdot \boldsymbol{n}=0$ is enforced on the surface represented by corresponding quadrature points in (c), where an additional flux is imposed on the immersed surface represented by the blue points.

Quadratic B-splines were used as the basis functions for the problem. The surface is represented by a fine triangulation generated and refined automatically from NETGEN [64], where surface quadrature points are obtained from a standard 6-point formula [65]. The code has been parallelized at the assembly level by OpenMP in the finite element tool FEAP [48, 66]. To test the scalability of the parallelization, four Newton iterations during the first time step of the chemomechanical problem shown in Figure 20 were computed. Figure 15 shows the overall time spent on the assembly for different numbers of parallel processes. We observe that a speedup ratio of 8 could be achieved with 12 processors.

Figure 16 shows the evolution of the concentration distribution. A core-shell structure is maintained during the period of the incoming flux. As a comparison, we also simulated the same problem with isogeometric analysis described in Section 4.3 . Figure 17 compares the concentration and the free energy distribution from the finite cell method with the isogeometric method at $t=1.34$. We observe that both methods yield a comparable concentration distribution. In addition, they also yield a comparable energy distribution, involving the first derivative of the primary degree of freedom.

\subsection{Mechanically supported diffusion and phase segregation in a composite electrode}

In the next step, we perform simulation of a mechanically coupled problem based on the formulation described in Section 5.1 and Section 5.2 .

Since there are two different materials in the problem, we use two background meshes, which are coupled at the interface $\Gamma^{*}$ as illustrated in Figure 18. The two background mesh do not necessarily to be identical. In this paper, for simplicity, we use two identical meshes.

The procedure for an adaptive generation of the quadrature points is the same as illustrated in Section 5.3 with the only exception that in this problem two sets of volume quadrature points need to be generated, as shown in Figure 19 


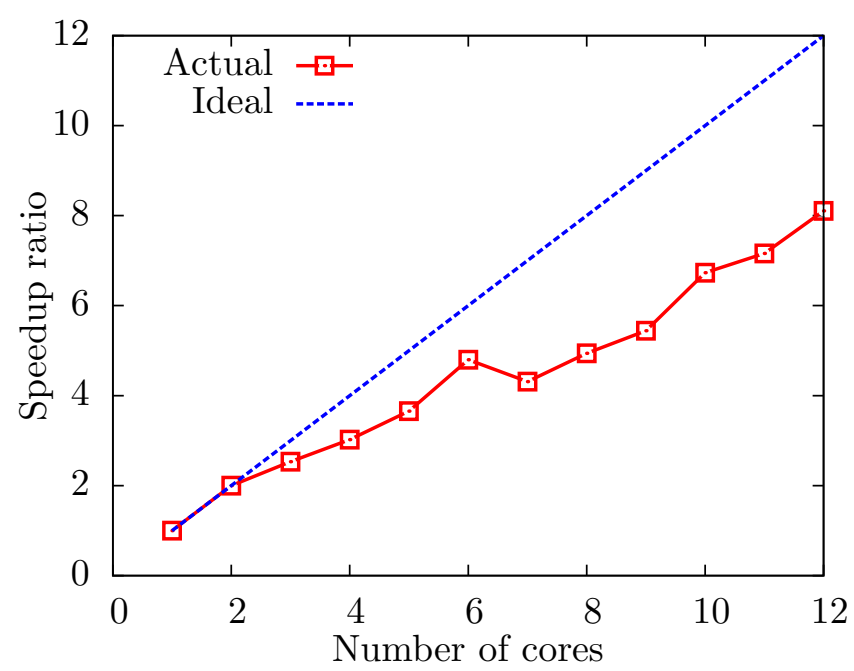

FIGURE 15: Speedup ratio of parallelization of the assembly of the global matrix by OpenMP. The computation was performed at the Lichtenberg cluster hpb-nodes at Technical University Darmstadt.

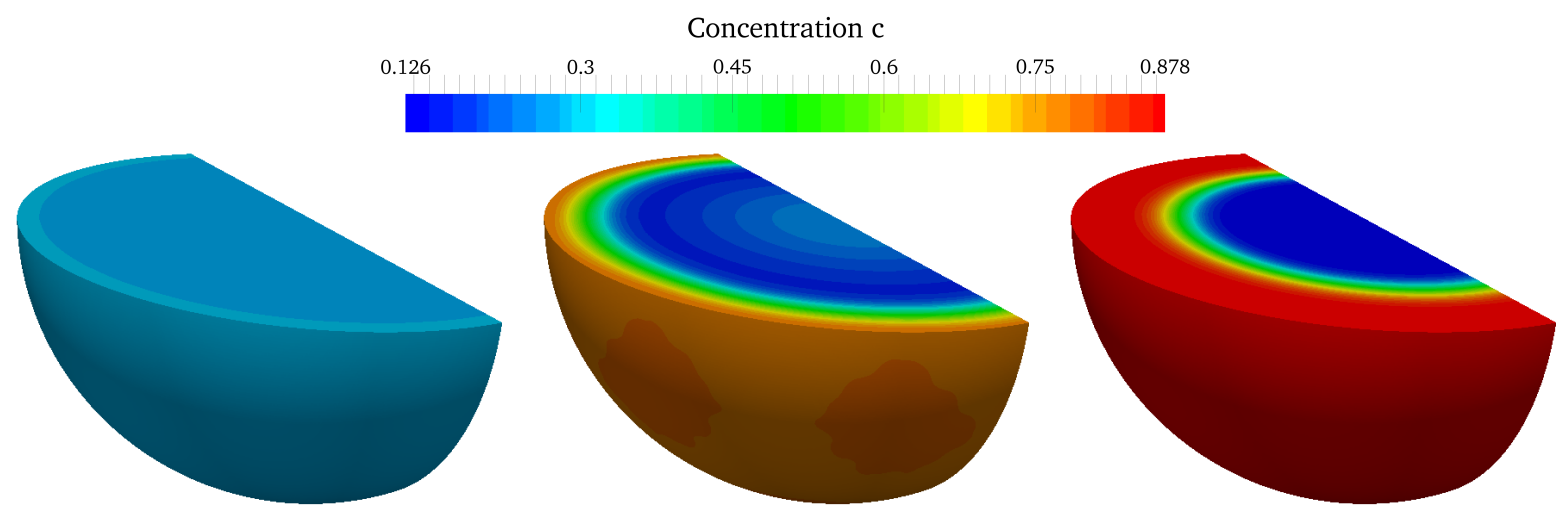

(a) $t=0.027$

(b) $t=0.445$

(c) $t=1.34$

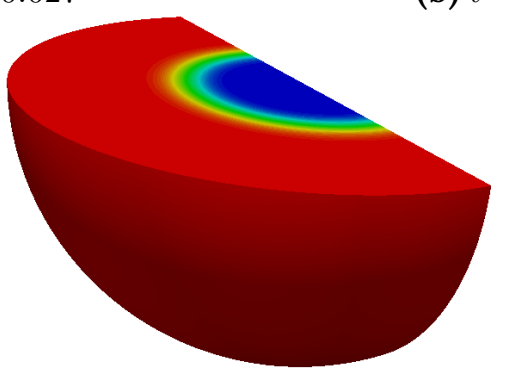

(d) $t=2.04$

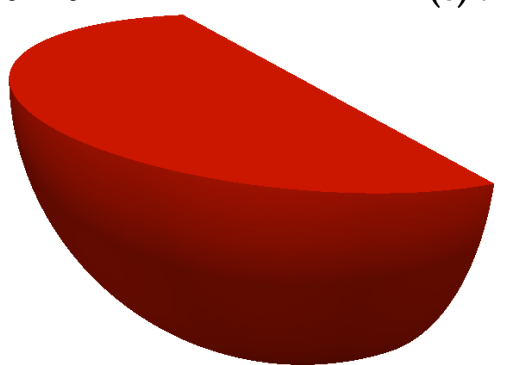

(e) $t=4.51$

FIGURE 16: Evolution of the concentration in a sphere with an incoming flux with finite cell method. 


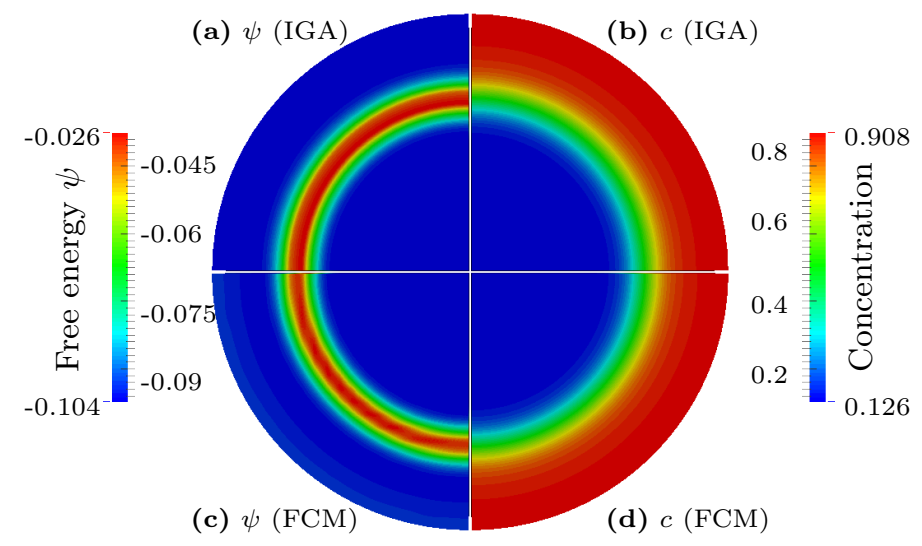

FIGURE 17: The contour plot of free energy $(a, c)$ and concentration $(b, d)$ simulated by IGA and the FCM, at $t=1.34$. For IGA, $10^{3}$ quadratic elements with NURBS basis functions are used; for FCM, $20^{3}$ elements with quadratic B-splines are used and the sub-cell level is 3. The concentration and free energy distributions obtained by the two methods are the same.

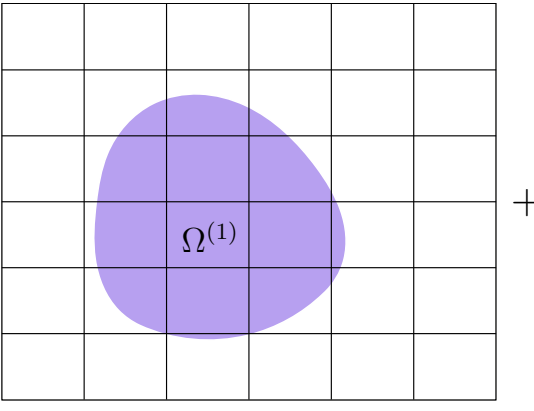

(a) $\boldsymbol{u}^{\mathrm{I}(1)}, c^{\mathrm{I}(1)}$

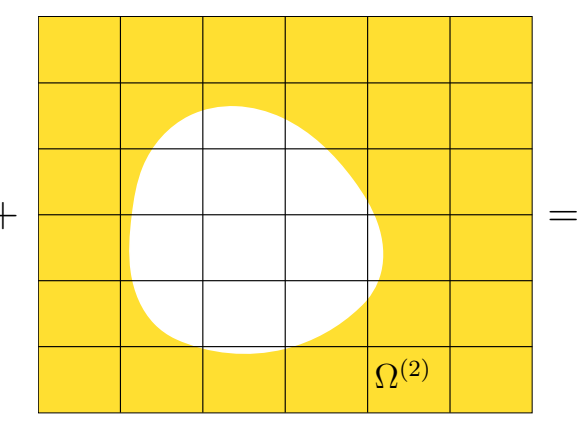

(b) $\boldsymbol{u}^{\mathrm{I}(2)}, c^{\mathrm{I}(2)}$

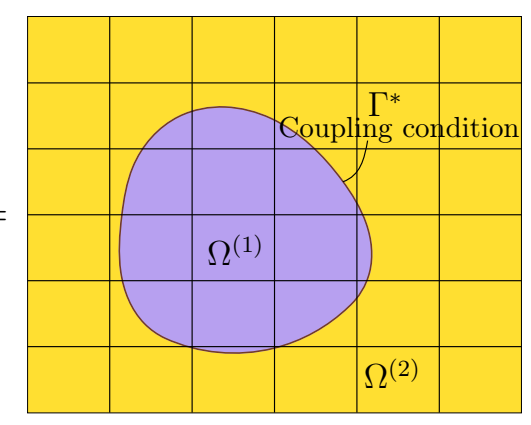

(c) $\boldsymbol{u}^{\mathrm{I}(1)}, c^{\mathrm{I}(1)}, \boldsymbol{u}^{\mathrm{I}(2)}, c^{\mathrm{I}(2)}$

FIGURE 18: Illustration of the coupling of two materials. The mesh (a) with the information of the particle $\left(\boldsymbol{u}^{\mathrm{I}(1)}, c^{\mathrm{I}(1)}\right)$ coincides with the mesh $(b)$ with the information of the matrix $\left(\boldsymbol{u}^{\mathrm{I}(2)}, c^{\mathrm{I}(2)}\right)$. The two meshes couple at the interface $\Gamma^{*}$ in Figure (c). In this paper, the two meshes are identical. 


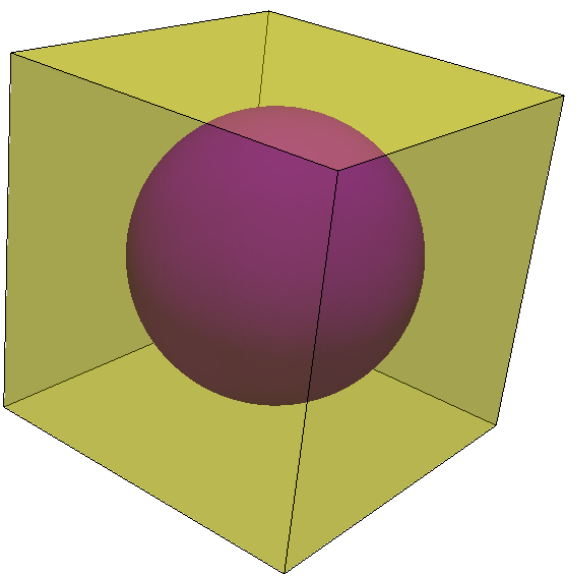

(a) Geometry

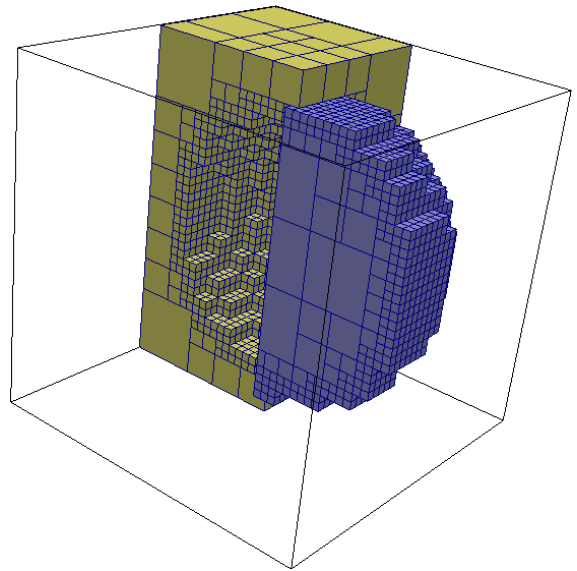

(b) Sub-cells

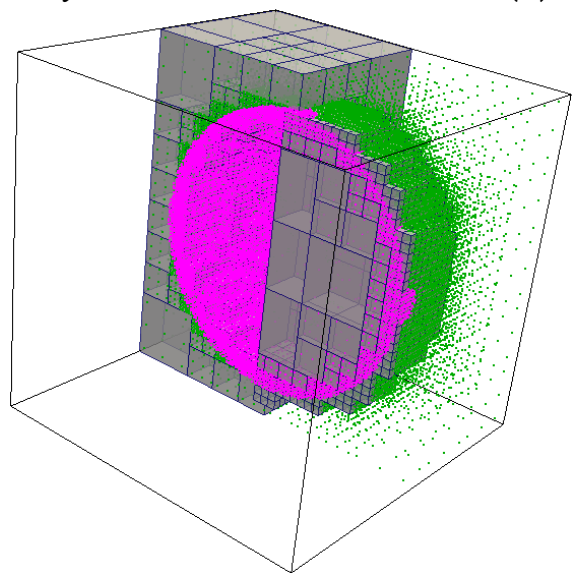

(c) Quadratures (V)

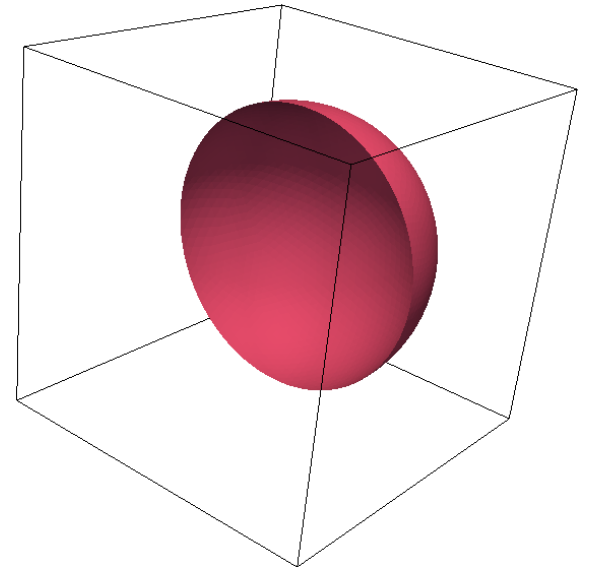

(d) Surface

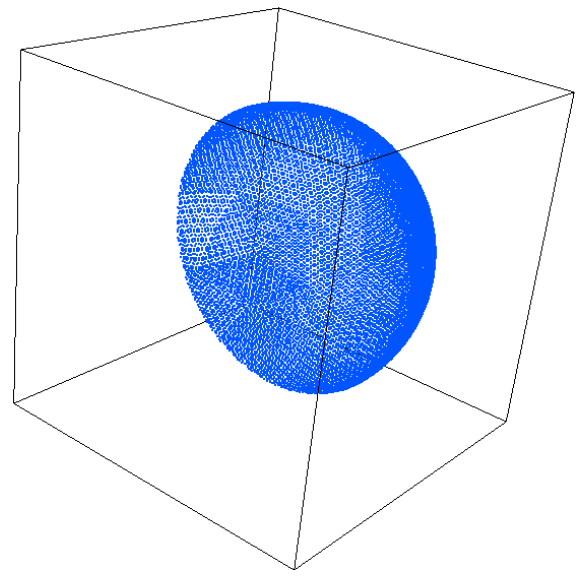

(e) Quadratures (S)

FIGURE 19: Work-flow of producing integration points for the volume and the interface of the composite electrode. The spherical particle is embedded in a cubic matrix (a). Based on the geometry, an adaptive subdivision of level 3 is performed for both the matrix and the particle (b). The corresponding Gaussian quadrature points are then produced (c). For the interface (d), the surface quadrature points are produced based on the surface mesh generated by NETGEN (e). 


\begin{tabular}{rccc}
\hline & Particle $\left(\Omega^{(1)}\right)$ & Matrix $\left(\Omega^{(2)}\right)$ & First appear in \\
\hline Bulk modulus (K) & 1 & 0.1 & Eq. (38) \\
Shear modulus (G) & 1 & 0.1 & Eq. (39) \\
Molar volume (V) & 0.08 & 0.0001 & Eq. (38) \\
Phase parameter $(\chi)$ & 2.5 & 1.0 & Eq. (41) and Eq. (42) \\
Interface parameter $(\kappa)$ & $1.247 \times 10^{-3}$ & - & Eq. (41) \\
Initial concentration $\left(c_{0}\right)$ & 0.25 & 0.9 & Eq. (43) and Eq. (44) \\
Boundary concentration $(\bar{c})$ & - & 0.9 & Eq. (50)
\end{tabular}

TABLE 3: Material parameters for the composite electrode. All parameters are given in dimensionless form.

The parameters for the simulation are listed in Table 3 The matrix is ten times softer than the particle. We apply an initial perturbing flux across the interface of 0.01 in the direction of the particle core, which is removed after 5 seconds.

Figure 20 -Figure 22 show the simulation results of composite electrodes with different embedding particles. The mechanical constraints are only applied on the matrix to prevent the rigid body movements (see Figure 20a). When the species transfer from the matrix to the particle, we observe only a slight gradient of the concentration field in the matrix, while more complex phenomena occur in the particles. For a spherical particle shown in the Figure 20, Li-rich phase islands start to form from the surface, surrounded by Li-deficit phase areas. Due to the unevenly distributed lithium concentration, the deformation of the particle is also inhomogeneous. During the period when the flux is applied, Li-rich phases merge into larger volumes.

We also simulate the same problem for geometrically more complicated electrode particles such as configurations based on two and three overlapping spheres (see Figures 21 and 22). These examples demonstrate the geometric flexibility of the finite cell method, which represents the geometry only via adaptive quadrature points. In both cases, the Li-rich phases emerge from the ends, since the compressive stresses arising around the bottlenecks drive the species towards the ends with tensile stresses. The Li-rich phases then marches towards the necks and coalesce. The remnant of Li-deficit phase is due to the incomplete charge. With longer incoming flux the particle can be finally fully charged.

Figure 23 shows the concentration distribution and the deformation of the matrix in the above three examples at $t=3.0$. A homogeneous Dirichlet boundary is prescribed on the outer surface and a small flux is given. The concentration variation is not large, which is very different from what has been observed in the particle, where the phase-segregation occurs. The unevenly distributed concentration profile on the interface is due to an uneven deformation and the consequent uneven stress distribution.

\section{Summary, conclusions, and outlook}

This paper deals with the formulation of variational boundary conditions for the CahnHilliard equation based on Nitsche's method, with a particular focus on applying this formulation in unfitted finite element discretizations such as the finite cell method. The Cahn-Hilliard equation describes the diffusion of species in materials that experience phase segregation. Since 

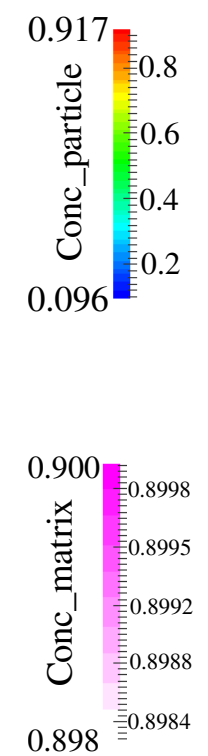

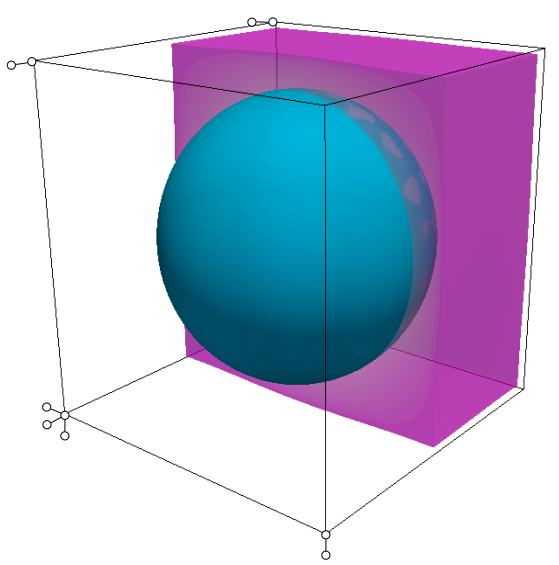

(a) $t=0$

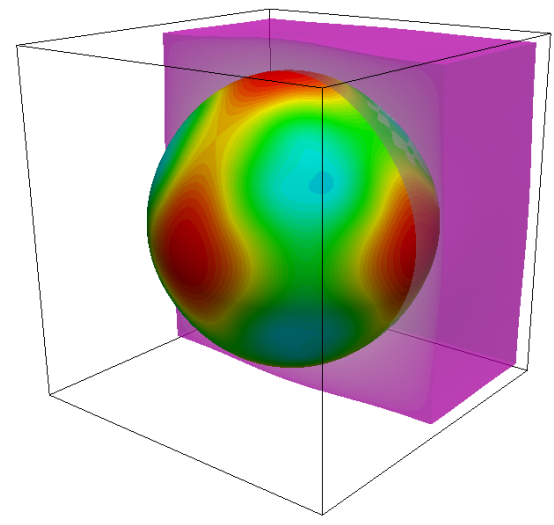

(c) $t=1.91$

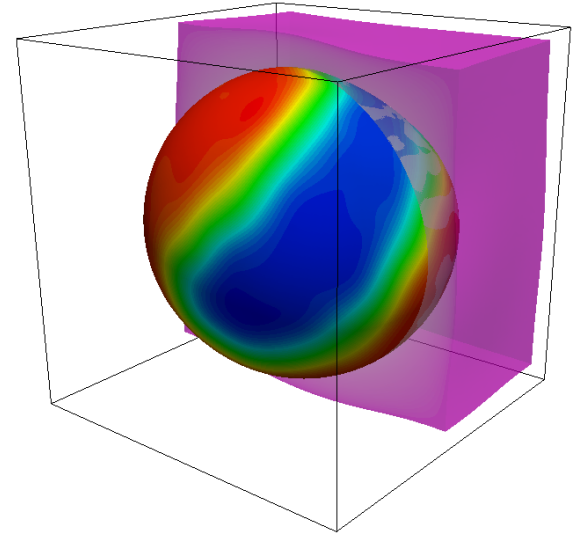

(e) $t=4.06$

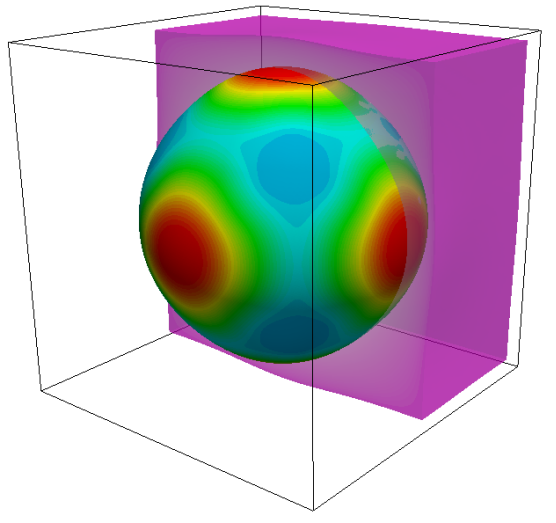

(b) $t=1.34$

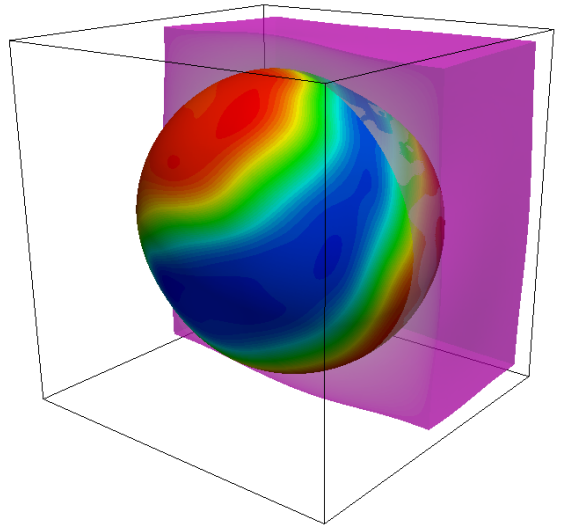

(d) $t=2.99$

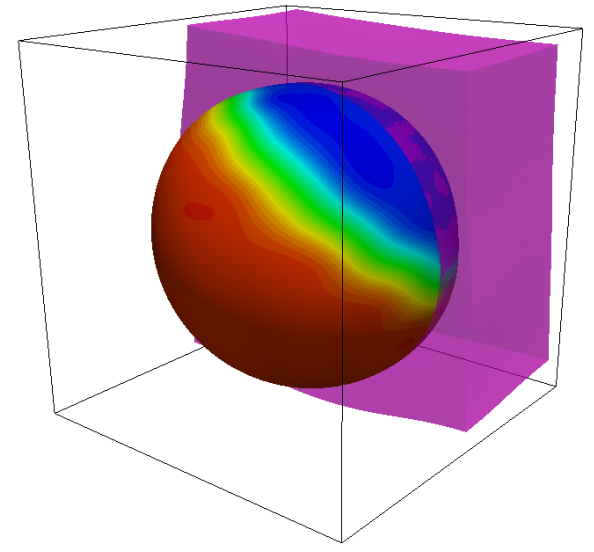

(f) $t=9.74$

FIGURE 20: Concentration distribution of the composite electrode with one sphere embedded in the matrix at different times. Minimum mechanical constraints are applied on the matrix to prevent the rigid body movements (a). After the flux flows into the particle, two phases emerge from the surface. As the flux comes in continuously, islands of Li-rich phases merge together before reaching the final state. 

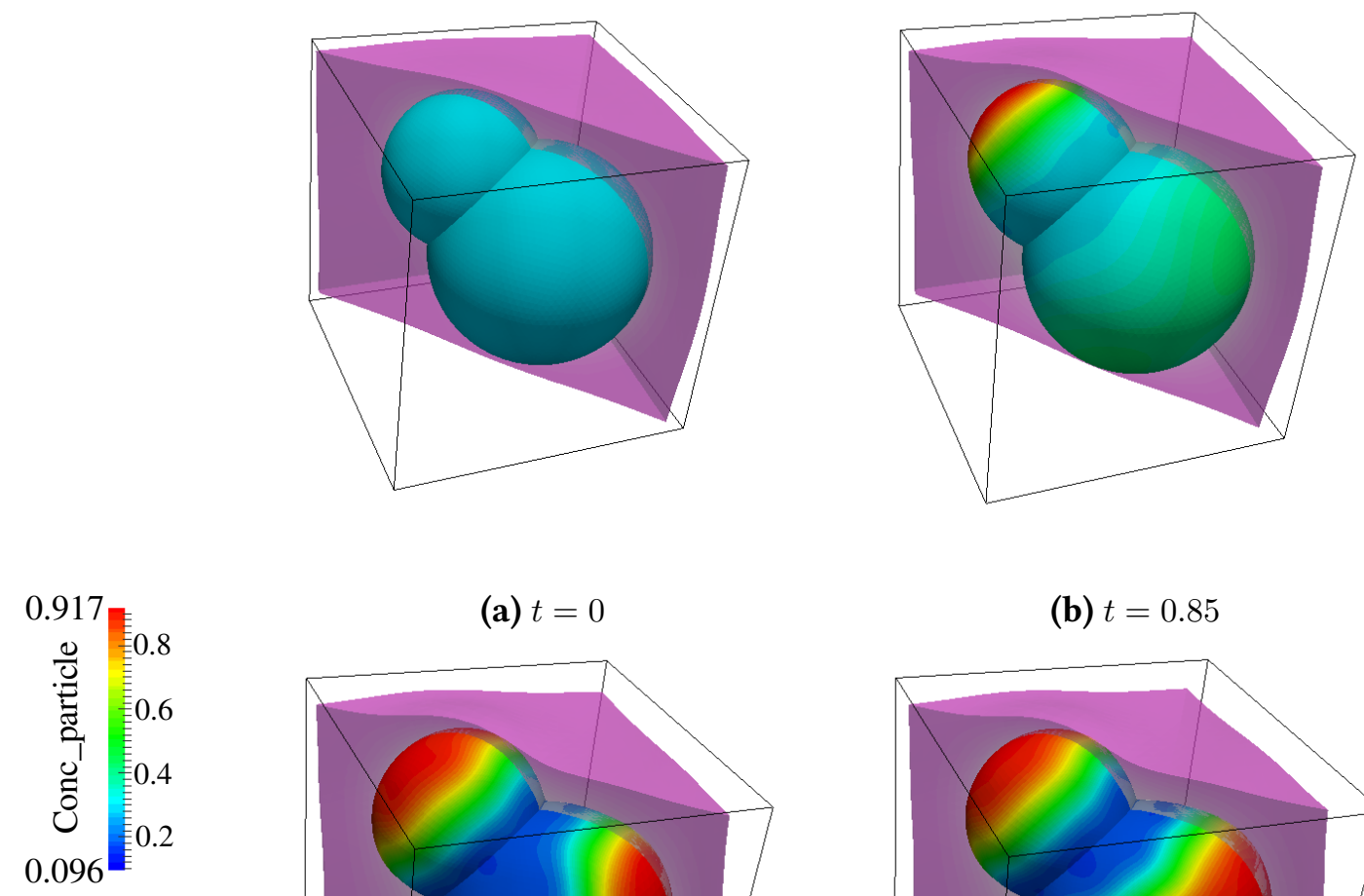

(a) $t=0$

(b) $t=0.85$
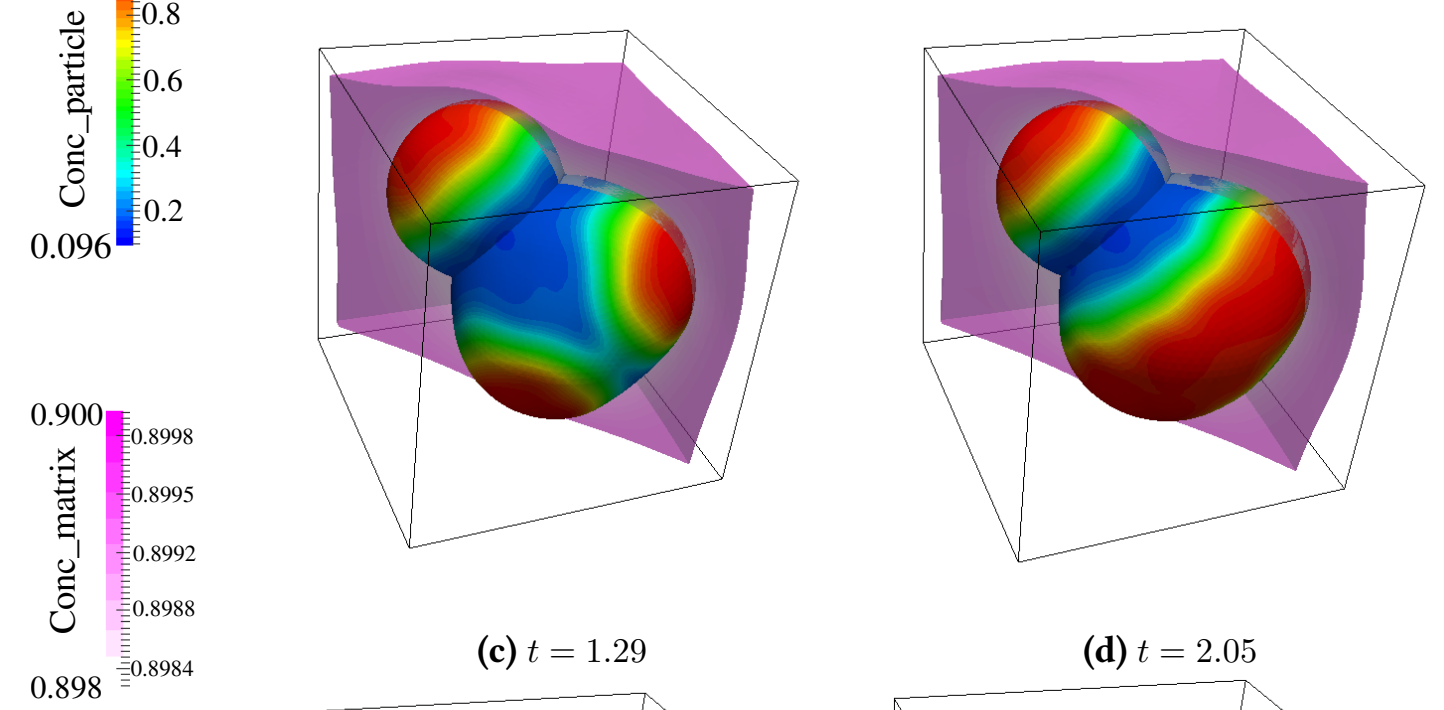

(c) $t=1.29$

(d) $t=2.05$
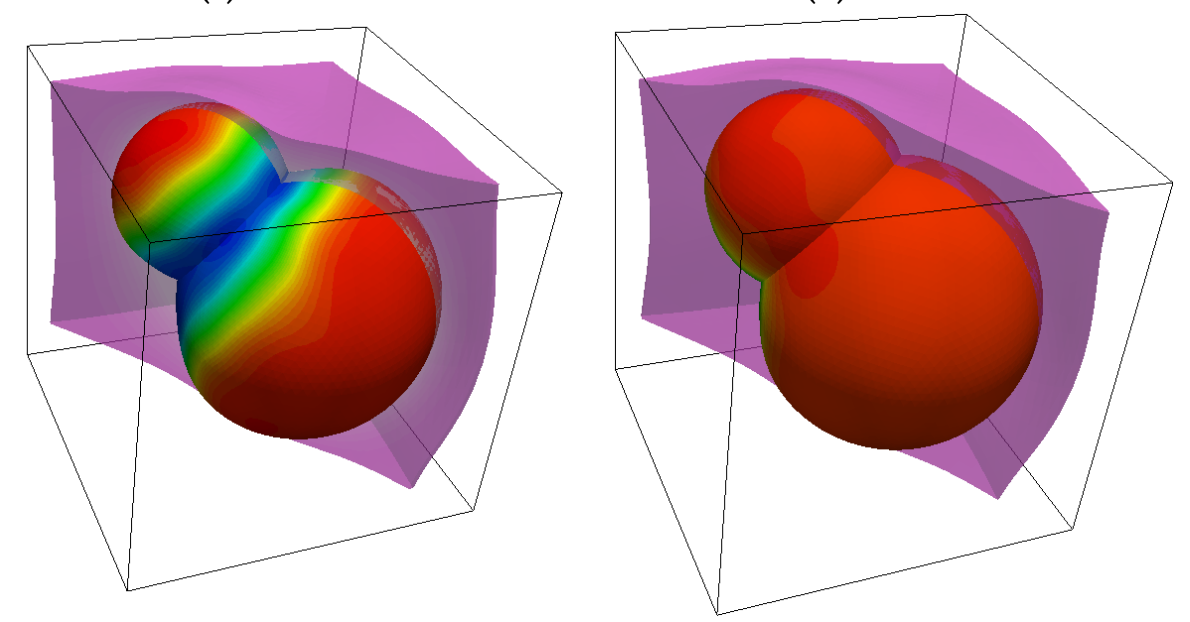

(e) $t=3.94$

(f) $t=31.13$

FIGURE 21: Concentration evolution of the composite electrode with two merged spheres embedded in the matrix. The Li-rich phases emerge from the two ends and finally join at the neck. 

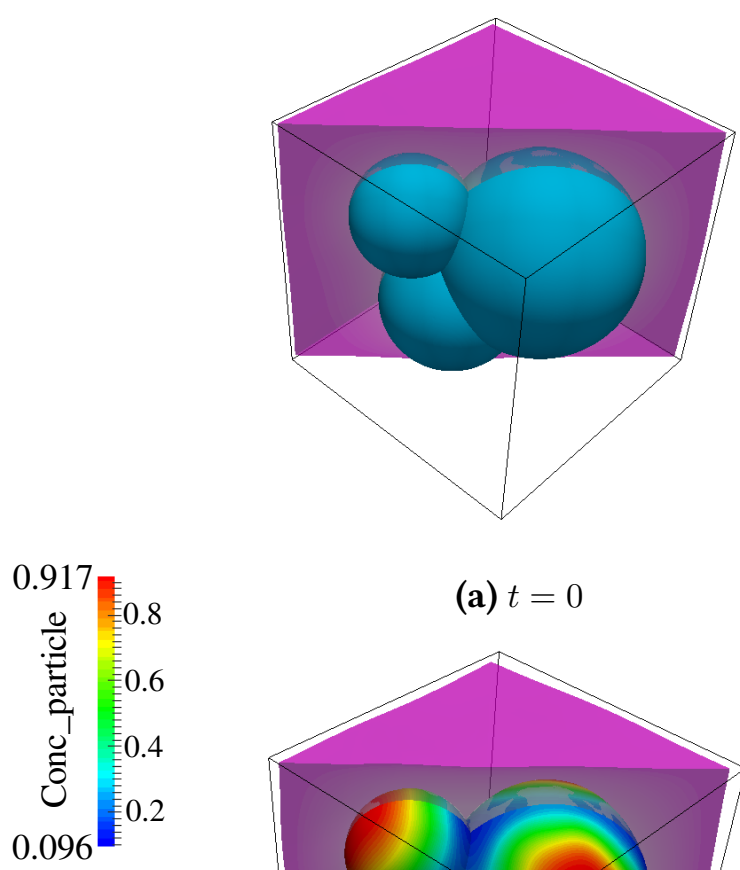

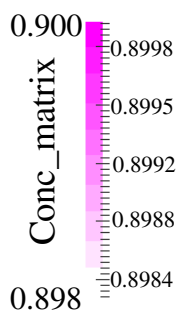

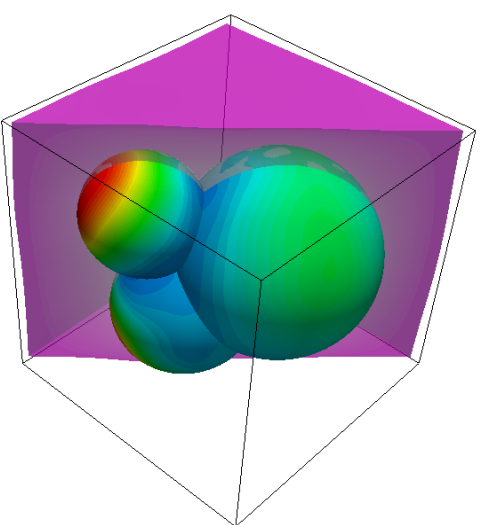

(b) $t=0.88$

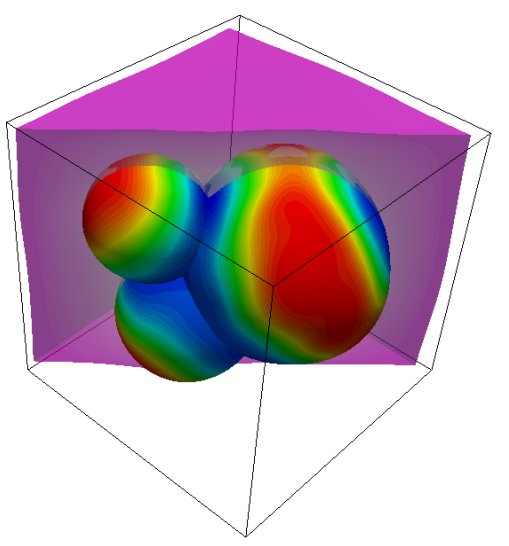

(d) $t=1.71$

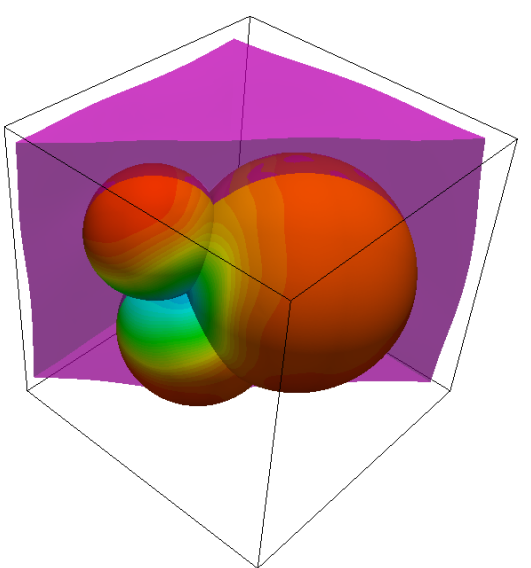

(f) $t=6.25$

FIGURE 22: Concentration evolution of the composite electrode with three merged spheres embedded in the matrix. Similar as the case with two particles in Figure 21. the Li-rich phases emerge from the three ends and finally integrate at the necks. 


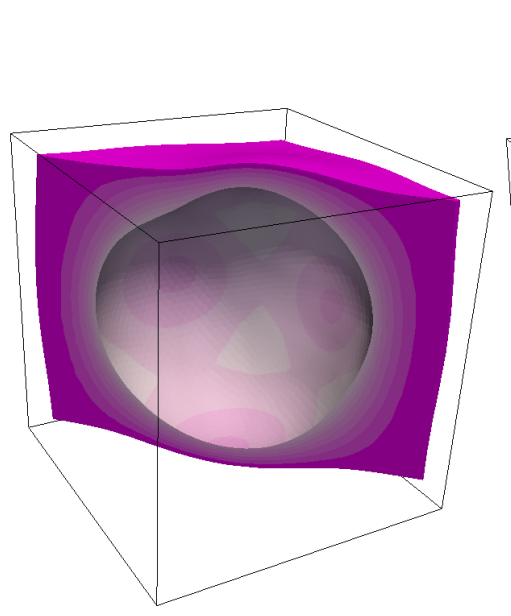

(a) Single sphere.

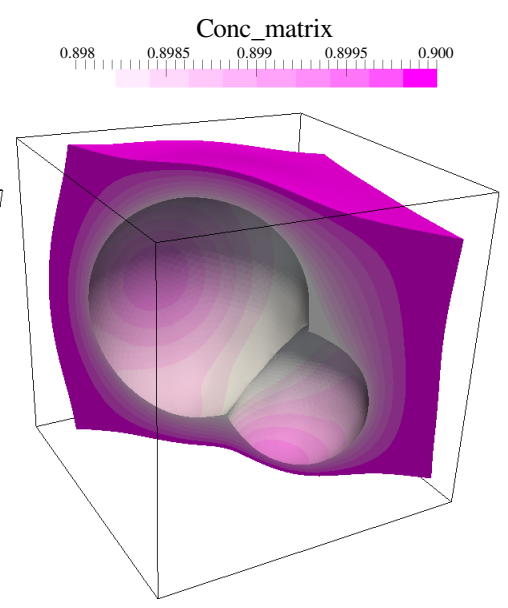

(b) Two spheres.

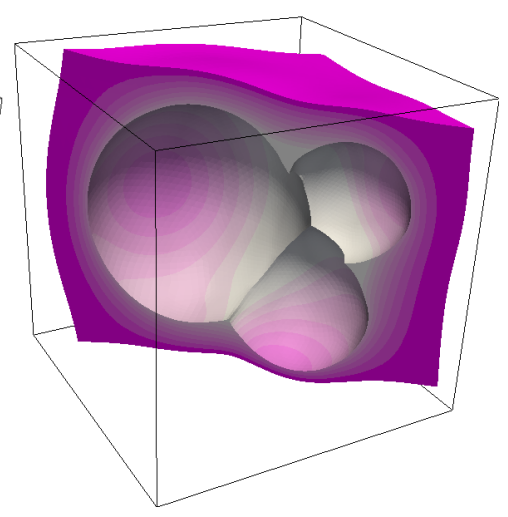

(c) Three spheres.

FIGURE 23: The concentration and deformation in the matrix at $t=3.00$ with different-shaped embedding particles. On the surface of the particle, a Dirichlet boundaryc $=0.9$ is prescribed, and only minimum points are constraint to prevent mechanical rigid body movements. On the interface, an out-going flux is given.

the Cahn-Hilliard equation is a fourth-order partial differential equation, shape functions that are globally $\mathscr{E}^{1}$-continuous are needed when a straightforward formulation based on primal unknowns is applied. This raises the questions of how to treat the additional boundary constraint $\boldsymbol{\nabla} c \cdot \boldsymbol{n}=0$, which in this paper is achieved by weak imposition based on Nitsche's method.

We successfully demonstrated the accuracy and flexibility of our formulation in the context of fitted and unfitted spline-based finite element methods. This includes a range of test examples in one, two and three dimensions, such as spinodal decomposition and a prolate spheroidal particle. The results showed that the Nitsche formulation is competitive with alternative method based on Lagrange multipliers and achieves optimal accuracy. Finally, we demonstrated the versatility of weak boundary and interface conditions in conjunction with the finite cell method. To this end, we computed phase segregation processes in a composite electrode, based on a mechanically coupled Cahn-Hilliard model. We showed that the finite cell method based on unfitted meshes yields the same accuracy than isogeometric analysis based on with geometrically exact boundary-fitted NURBS discretizations. We then illustrated that the finite cell method enables the analysis of geometrically complex particle shapes, which is hardly possible with boundaryfitted isogeometric analysis. From a modeling point of view, our results show that the mechanical stresses influence the phase segregation in the particle. In particular, we observed that the flux flows from areas with tensile stresses towards areas with compressions.

Future work will transfer the technology described in this paper to the analysis of real-world particle configurations, whose geometry will be extracted from diagnostic imaging data such as high-resolution computed tomography scans. 


\section{Acknowledgments}

This work is supported by the "Excellence Initiative" of the German Federal and State Governments and the Graduate School of Computational Engineering at the Technische Universität Darmstadt. The authors gratefully acknowledge the computing time granted on the Hessian High Performance Computer "Lichtenberg". D. Schillinger gratefully acknowledges support from the National Science Foundation through grant agreement CISE-1565997 and the NSF CAREER Award No. 1651577. B. Xu would particularly like to thank the Adolf Messer Foundation for awarding her the Adolf Messer Prize and for the financial support. (2015) 325-347.

[12] Y. Zhao, B.-X. Xu, P. Stein, D. Gross, Phase-field study of electrochemical reactions at exterior and interior

[13] H. Gómez, A. Reali, G. Sangalli, Accurate, efficient, and (iso)geometrically flexible collocation methods for phase-field models, Journal of Computational Physics 262 (2014) 153-171.

[14] L. Dalcin, N. Collier, P. Vignal, A. M. A. Côrtes, V. M. Calo, PetIGA: A framework for high-performance isogeometric analysis, Computer Methods in Applied Mechanics and Engineering 308 (2016) 151-181.

[15] S. Kaessmair, P. Steinmann, Comparative computational analysis of the Cahn-Hilliard equation with emphasis on $C^{1}$-continuous methods, Journal of Computational Physics 322 (2016) 783-803.

[16] D. Anders, C. Hesch, K. Weinberg, Computational modeling of phase separation and coarsening in solder alloys, International Journal of Solids and Structures 49 (2012) 1557-1572.

[17] J. Nitsche, Über ein Variationsprinzip zur Lösung von Dirichlet-Problemen bei Verwendung von Teilräumen, die keinen Randbedingungen unterworfen sind, Abhandlungen aus dem Mathematischen Seminar der Universität Hamburg 36 (1970) 9-15. 
[18] M. Griebel, M. Schweitzer, A particle-partition of unity method. Part V: boundary conditions., in: S. Hildebrandt, H. Karcher (Eds.), Geometric Analysis and Nonlinear Partial Differential Equations, Springer, 2004, pp. 519-542.

[19] A. Hansbo, P. Hansbo, An unfitted finite element method, based on Nitsche's method, for elliptic interface problems, Computer Methods in Applied Mechanics and Engineering 191 (2002) 537-552.

[20] Y. Bazilevs, T. Hughes, Weak imposition of Dirichlet boundary conditions in fluid mechanics, Computers \& Fluids 36 (2007) 12-26.

[21] J. Evans, T. Hughes, Explicit trace inequalities for isogeometric analysis and parametric hexahedral finite elements, Numerische Mathematik 123 (2013) 259-290.

[22] D. Schillinger, M. Ruess, N. Zander, Y. Bazilevs, A. Düster, E. Rank, Small and large deformation analysis with the $p$ - and B-spline versions of the Finite Cell Method, Computational Mechanics 50(4) (2012) 445-478.

[23] D. Schillinger, M. Ruess, The Finite Cell Method: A review in the context of higher-order structural analysis of CAD and image-based geometric models, Archives of Computational Methods in Engineering 22(3) (2015) 391-455.

[24] A. Embar, J. Dolbow, I. Harari, Imposing Dirichlet boundary conditions with Nitsche's method and spline-based finite elements, International Journal for Numerical Methods in Engineering 83 (2010) 877-898.

[25] I. Harari, E. Grosu, A unified approach for embedded boundary conditions for fourth-order elliptic problems, International Journal for Numerical Methods in Engineering 104 (2015) 655-675.

[26] W.-J. Zhang, Lithium insertion/extraction mechanism in alloy anodes for lithium-ion batteries, Journal of Power Sources 196 (2011) 877-885.

[27] C. Delmas, M. Maccario, L. Croguennec, F. Le Cras, F. Weill, Lithium deintercalation in $\mathrm{LiFePO}_{4}$ nanoparticles via a domino-cascade model, Nature Materials 7 (2008) 665-671.

[28] E. Guggenheim, Mixtures: The Theory of the Equlibrium Properties of Some Simple Classes of Mixtures Solutions and Alloys, The International series of monographs on physics, Clarendon Press, 1952. URL: https : //books.google.de/books?id=1tXQAAAAMAAJ

[29] M. Ruess, D. Schillinger, A. Özcan, E. Rank, Weak coupling for isogeometric analysis of non-matching and trimmed multi-patch geometries, Computer Methods in Applied Mechanics and Engineering 269 (2014) 4671.

[30] J. Cottrell, T. Hughes, Y. Bazilevs, Isogeometric analysis: Towards Integration of CAD and FEA, John Wiley \& Sons, 2009.

[31] D. Grossmann, B. Jüttler, H. Schlusnus, J. Barner, A. Vuong, Isogeometric simulation of turbine blades for aircraft engines, Computer Aided Geometric Design 29(7) (2012) 519-531.

[32] D. Schillinger, J. Evans, A. Reali, M. Scott, T. Hughes, Isogeometric collocation: Cost comparison with Galerkin methods and extension to adaptive hierarchical NURBS discretizations, Computer Methods in Applied Mechanics and Engineering 267 (2013) 170-232.

[33] J. Cottrell, A. Reali, Y. Bazilevs, T. Hughes, Isogeometric analysis of structural vibrations, Computer Methods in Applied Mechanics and Engineering 195 (2006) 5257-5296.

[34] T. Hughes, A. Reali, G. Sangalli, Duality and unified analysis of discrete approximations in structural dynamics and wave propagation: Comparison of $p$-method finite elements with $k$-method NURBS, Computer Methods in Applied Mechanics and Engineering 197 (2008) 4104-4124.

[35] T. Hughes, J. Evans, A. Reali, Finite element and NURBS approximations of eigenvalue, boundary-value, and initial-value problems, Computer Methods in Applied Mechanics and Engineering 272 (????) 290-320.

[36] C. Verhoosel, M. Scott, M. Borden, T. Hughes, R. de Borst, Discretization of higher-order gradient damage models using isogeometric finite elements, Computer Methods in Applied Mechanics and Engineering 197 (2008) 2976-2988.

[37] J. Kiendl, F. Auricchio, T. Hughes, A. Reali, Single-variable formulations and isogeometric discretizations for shear deformable beams, Computer Methods in Applied Mechanics and Engineering 284 (2015) 988-1004.

[38] L. Piegl, W. Tiller, The NURBS Book, Springer, 1997.

[39] M. Borden, M. Scott, J. Evans, T. Hughes, Isogeometric finite element data structures based on Bézier extraction of NURBS, International Journal for Numerical Methods in Engineering 87 (2011) 15-47.

[40] D. Schillinger, P. Ruthala, L. Nguyen, Lagrange extraction and projection for spline basis functions: a direct 
link between isogeometric and standard nodal finite element formulations, International Journal for Numerical Methods in Engineering doi:10.1002/nme.5216 (2016).

[41] J. Liu, C. Landis, H. Gomez, T. Hughes, Liquid-vapor phase transition: Thermomechanical theory, entropy stable numerical formulation, and boiling simulations, Computer Methods in Applied Mechanics and Engineering 297 (2015) 476-553.

[42] R. Cools, Monomial cubature rules since "Stroud": A compilation - Part 2, Journal of Computational and Applied Mathematics 112 (1999) 21-27.

[43] C. Felippa, Advanced Finite Element Methods, 2013. URL: http://WwW. colorado.edu/ engineering/CAS/courses.d/AFEM.d/Home.html. [Lecture notes].

[44] A. Düster, J. Parvizian, Z. Yang, E. Rank, The finite cell method for three-dimensional problems of solid mechanics, Computer Methods in Applied Mechanics and Engineering 197 (2008) 3768-3782.

[45] F. Xu, D. Schillinger, D. Kamensky, V. Varduhn, C. Wang, M.-C. Hsu, The tetrahedral finite cell method for fluids: Immersogeometric analysis of turbulent flow around complex geometries, Computers \& Fluids doi:10.1016/j.compfluid.2015.08.027 (2016).

[46] V. Varduhn, M.-C. Hsu, M. Ruess, D. Schillinger, The tetrahedral finite cell method: Higher-order immersogeometric analysis on adaptive non-boundary-fitted meshes, International Journal for Numerical Methods in Engineering doi:10.1002/nme.5207 (2016).

[47] D. Schillinger, L. Dedè, M. Scott, J. Evans, M. Borden, E. Rank, T. Hughes, An isogeometric design-throughanalysis methodology based on adaptive hierarchical refinement of NURBS, immersed boundary methods, and T-spline CAD surfaces, Computer Methods in Applied Mechanics and Engineering 249-250 (2012) 116-150.

[48] R. L. Taylor, FEAP - Finite Element Analysis Program, 2014. URL: http : //www . ce.berkeley . edu/ projects/feap

[49] P. Stein, B. Xu, 3D Isogeometric Analysis of intercalation-induced stresses in Li-ion battery electrode particles, Computer Methods in Applied Mechanics and Engineering 268 (2014) 225-244.

[50] B. Kang, G. Ceder, Battery materials for ultrafast charging and discharging, Nature 458 (2009) 190-193.

[51] J. Li, D.-B. Le, P. P. Ferguson, J. R. Dahn, Lithium polyacrylate as a binder for tin-cobalt-carbon negative electrodes in lithium-ion batteries, Electrochimica Acta 55 (2010) 2991-2995.

[52] G. Liu, S. Xun, N. Vukmirovic, X. Song, P. Olalde-Velasco, H. Zheng, V. S. Battaglia, L. Wang, W. Yang, Polymers with Tailored Electronic Structure for High Capacity Lithium Battery Electrodes, Advanced Materials 23 (2011) 4679-4683.

[53] G. Liu, H. Zheng, X. Song, V. S. Battaglia, Particles and Polymer Binder Interaction: A Controlling Factor in Lithium-Ion Electrode Performance, Journal of The Electrochemical Society 159 (2012) A214-A221.

[54] M. Doyle, T. F. Fuller, J. Newman, Modeling of Galvanostatic Charge and Discharge of the Lithium/Polymer/Insertion Cell, Journal of The Electrochemical Society 140 (1993) 1526-1533.

[55] A. Bower, P. Guduru, V. Sethuraman, A finite strain model of stress, diffusion, plastic flow, and electrochemical reactions in a lithium-ion half-cell, Journal of the Mechanics and Physics of Solids 59 (2011) 804-828.

[56] A. Salvadori, D. Grazioli, M. G. D. Geers, Governing equations for a two-scale analysis of Li-ion battery cells, International Journal of Solids and Structures 59 (2015) 90-109.

[57] S. Lee, A. M. Sastry, J. Park, Study on microstructures of electrodes in lithium-ion batteries using variational multi-scale enrichment, Journal of Power Sources 315 (2016) 96-110.

[58] S. A. Roberts, V. E. Brunini, K. N. Long, A. M. Grillet, A Framework for Three-Dimensional Mesoscale Modeling of Anisotropic Swelling and Mechanical Deformation in Lithium-Ion Electrodes, Journal of The Electrochemical Society 161 (2014) F3052-F3059.

[59] H. Mendoza, S. A. Roberts, V. E. Brunini, A. M. Grillet, Mechanical and Electrochemical Response of a LiCoO2 Cathode using Reconstructed Microstructures, Electrochimica Acta 190 (2016) 1-15.

[60] D. R. Noble, E. P. Newren, J. B. Lechman, A conformal decomposition finite element method for modeling stationary fluid interface problems, International Journal for Numerical Methods in Fluids 63 (2010) 725-742.

[61] B. Orvananos, H.-C. Yu, A. Abdellahi, R. Malik, C. P. Grey, G. Ceder, K. Thornton, Kinetics of Nanoparticle Interactions in Battery Electrodes, Journal of The Electrochemical Society 162 (2015) A965-A973.

[62] P. Stein, Y. Zhao, B.-X. Xu, Effects of surface tension and electrochemical reactions in Li-ion battery electrode nanoparticles, Journal of Power Sources 332 (2016) 154-169. 
[63] D. Schillinger, I. Harari, M.-C. Hsu, D. Kamensky, K. F. Stoter, Y. Yu, Y. Zhao, The non-symmetric nitsche method for the parameter-free imposition of weak boundary and coupling conditions in immersed finite elements, Computer Methods in Applied Mechanics and Engineering 309 (2016) 625-652.

[64] J. Schöberl, NETGEN An advancing front 2d/3d-mesh generator based on abstract rules, Computing and Visualization in Science 1 (1997) 41-52.

[65] T. J. R. Hughes, The Finite Element Method: Linear Static and Dynamic Finite Element Analysis, Dover, 2000, pp. 109-184.

[66] P. Jarzebski, K. Wisniewski, R. L. Taylor, On parallelization of the loop over elements in FEAP, Computational Mechanics 56 (2015) 77-86. 